\title{
Mild deficiency of mitochondrial Complex III in a mouse model of Alzheimer's disease decreases amyloid beta plaque formation
}

milena pinto ( $\sim$ mpinto@med.miami.edu )

University of Miami School of Medicine https://orcid.org/0000-0001-9202-1527

Francisca Diaz

University of Miami Miller School of Medicine: University of Miami School of Medicine

Nadee Nissanka

University of Miami Miller School of Medicine: University of Miami School of Medicine

Chelsey S Guastucci

University of Miami Miller School of Medicine: University of Miami School of Medicine

Placido Illiano

University of Miami Miller School of Medicine: University of Miami School of Medicine

Roberta Brambilla

University of Miami Miller School of Medicine: University of Miami School of Medicine

Carlos T Moraes

University of Miami Miller School of Medicine: University of Miami School of Medicine

\section{Research article}

Keywords: Mitochondria dysfunctions, Alzheimer's disease, mouse model, Oxidative stress

Posted Date: May 13th, 2021

DOl: https://doi.org/10.21203/rs.3.rs-500142/v1

License: (9) This work is licensed under a Creative Commons Attribution 4.0 International License.

Read Full License 


\section{Mild deficiency of mitochondrial Complex III in a mouse model of Alzheimer's disease decreases amyloid beta plaque formation.}

Milena Pinto ${ }^{1 *}$, Francisca Diaz¹, Nadee Nissanka ${ }^{1}$, Chelsey S. Guastucci², Placido lliano ${ }^{2}$, Roberta Brambilla ${ }^{2}$, Carlos T. Moraes ${ }^{1 *}$

Pinto, Milena <mpinto@med.miami.edu $>$

Francisca, Diaz< <diaz@med.miami.edu $>$

Nissanka, Nadee <n.nissanka@med.miami.edu>

Guastucci, S. Chelsey<css98@@miami.edu>

Illiano, Placido <pxi44@med.miami.edu>

Brambilla, Roberta <RBrambilla@med.miami.edu $>$

Moraes, Carlos T. <cmoraes@med.miami.edu>

*Correspondence: $\underline{\text { mpinto@med.miami.edu or cmoraes@med.miami.edu }}$

${ }^{1}$ Department of Neurology, University of Miami Miller School of Medicine, Miami, FL, USA

${ }^{2}$ The Miami Project to Cure Paralysis, Department of Neurological Surgery, University of Miami Miller School of Medicine, FL, USA 


\begin{abstract}
Background: For decades, mitochondrial dysfunctions and the generation of reactive oxygen species have been proposed to promote the development and progression of the amyloid pathology in Alzheimer's disease, but this association is still debated. In particular, it is still unclear if mitochondrial dysfunctions are a trigger or rather a consequence of the formation of amyloid aggregates, and in particular, the role of the different mitochondrial oxidative phosphorylation complexes in Alzheimer's patients' brain remains poorly understood.

Methods: To study how mitochondrial Complex III defects affect amyloid beta pathology in vivo, we partially knocked out mitochondrial Complex III $\left(\mathrm{CII}{ }^{\mathrm{KO}}\right)$ in mature forebrain neurons of an Alzheimer's mouse model that develops plaque pathology (APP/PS1).

Results: We found that Complex III dysfunction in adult neurons induced mild oxidative stress which did not correlate with increased amyloid beta accumulation. In fact, $\mathrm{CIII}{ }^{\mathrm{KO}}-\mathrm{AD}$ mice showed decreased plaque number, decreased $A \beta 42$ toxic fragment and altered amyloid precursor protein cleavage pathway.

Conclusions: Our results support a model in which mitochondrial dysfunction is not the cause of amyloid oligomer accumulation but rather a consequence of amyloid beta toxicity.
\end{abstract}

\title{
Keywords
}

Mitochondria dysfunctions, Alzheimer's disease, mouse model, Oxidative stress

\section{Background}

Alzheimer's disease (AD) is the most common cause of dementia and decline in memory and cognition in the elders. It is an age-related neurodegenerative disease characterized by the presence of extracellular amyloid plaques in cortex and hippocampus, mainly composed of amyloid beta $(A \beta)$ fragment, and neurofibrillary tangles, caused by intracellular accumulation of hyperphosphorylated Tau protein (1). Mitochondrial dysfunctions have been implicated in the development and progression of many neurodegenerative diseases, including $A D$, and a "mitochondrial hypothesis" has been proposed in which changes in mitochondrial function initiate the cascade that leads to the pathological hallmarks characteristic of $A D$, in particular to the formation of amyloid plaques (2). The most relevant alterations include oxidative phosphorylation (OXPHOS) defects, elevated oxidative stress, perturbations in mitochondrial dynamics and mitophagy, alterations in mitochondrial transport, increased mitochondrial DNA (mtDNA) mutations, and defective calcium homeostasis (3). Aging, the primary risk factor in 
$A D(4)$, is also associated with declines of mitochondrial function in the central nervous system (5-9). Accumulated mutations in mtDNA, which encodes proteins for several OXPHOS complexes, have been found in post mortem brains of AD patients (10-12) which also show decreased gene expression of all mtDNA-encoded genes and of the master mitochondrial biogenesis regulator, PGC-1a (13). Early studies that report defects in OXPHOS in AD patients (14-17) describe, in particular, deficiency of cytochrome c oxidase (Complex IV of the electron transport chain), at least partially responsible for increase in reactive oxygen species (ROS) production, decrease in energy stores, and disruption of energy metabolism $(18,19)$.

Scarcer are data on the other complexes and in particular of Complex III. Decreased Complex III core protein 1 was observed in the temporal cortex of patients with $A D(20)$, mitochondrial preparations of frontal cortex of $A D$ patients show deficiencies of Complex II, III and IV but no differences in respiratory supercomplexes (21). Other reports show how Complex III activity and Complex III/ Citrate Synthase (CIII/CS) ratio are higher in AD patients' platelets (22).

Despite years of study, the role of the different mitochondrial OXPHOS complexes in the brain of AD patients remains poorly understood. In particular, it is still unclear if mitochondrial dysfunctions are a trigger or rather a consequence of AD pathology. ROS can affect amyloid pathology by enhancing beta-secretase and gammasecretase activity and exacerbating amyloid beta fragment $(A \beta)$ production and aggregation (23-26), but amyloid pathology, on the other side, can also negatively affect mitochondrial function. $A \beta$ directly associates with mitochondria, inhibits OXPHOS (15, 27-33), and can alter fission and fusion processes (34).

We previously reported that severe isolated Complex IV deficiency led to a reduction in plaque formation and $A \beta$ steady-state levels in the APP/PS1 mouse model of AD (35) and that depletion of mtDNA also led to a decrease in plaques in the same model (36).

These findings were surprising and raised the question that severe OXPHOS defects may not reflect age related OXPHOS decay, which are mild. To directly analyze the contribution of a mild Complex III deficiency to the amyloid pathology, we crossed APP/PS1 AD transgenic mice with a RISP neuron specific conditional KO in which the assembly and function of mitochondrial Complex III is impaired in a subset of post-mitotic neurons, particularly CamKIla+ neurons in the cerebral cortex and the hippocampus. 


\section{Methods}

\section{Mouse Procedures}

The AD transgenic mice carry a mutant $\mathrm{APP}^{\mathrm{Swe}}$ and a mutant PSEN1 ${ }^{\mathrm{dE9}}$ allele (The Jackson Laboratory, Stock \#34832) (37), CaMKIla-CreERT2 transgenic mice express a tamoxifen-inducible Cre recombinase under the control of the mouse calcium/calmodulin-dependent protein kinase II alpha (CaMKIla) promoter region (38) (The Jackson Laboratory, Stock \#012362). Mice were crossed with RISPFF mice where exon 2 of the RISP gene is flanked by two loxP sites (39). Tamoxifen was prepared by first dissolving the powder in ethanol (20 mg per 100 $\mu \mathrm{l})$ and mixing this solution with $900 \mu \mathrm{l}$ corn oil for a final concentration of $20 \mathrm{mg} / \mathrm{ml}$. 5-month-old AD-RISPKO mice $\left(\mathrm{RISPFF}_{-} \mathrm{AD}^{+/-}-\mathrm{CaMKIlaCreERT2}{ }^{+/}\right)$and control mice $\left(\mathrm{RISPFF}_{-} \mathrm{AD}^{+/}\right)$were injected IP with $130 \mathrm{mg}$ per kilogram body weight of tamoxifen once a day for 5 consecutive days. All experiments and animal husbandry were performed according to a protocol approved by the University of Miami Institutional Animal Care and Use Committee. Mice were housed in a virus-antigen-free facility of the University of Miami, Division of Veterinary Resources in a 12-h light/dark cycle at room temperature and fed ad libitum.

\section{Quantitative PCR}

Total DNA was extracted from the cortex and hippocampus using standard phenol/chloroform extraction. Quantitative PCR reactions done using SYBR chemistry (SsoAdvanced Universal Master Mix SYBR Green, BioRad) were performed on a Bio-Rad CFX96/C1000 qPCR machine. The comparative ddCt method was used to determine the relative levels of undeleted and deleted UQCRFS1 to a control genomic region (40). To estimate the levels of "undeleted UQCRFS1" we designed primers to amplify a region in Exon 2 of levels UQCRFS1 (F:AACCAAGATGAGTACAGACA, R:AGAACCAAGAAGGAGATTGA); to estimate the levels of "deleted UQCRFS1" we designed primers in the intron sequences flanking Exon 2 of UQCRFS1 (F:TCATCCGAGACCCAGCAA, R:AGCACATAGCAGAGATACAA). Primers for beta-Actin were (F:GCGCAAGTACTCTGTGTGGA, R:CATCGTACTCCTGCTTGCTG).

\section{Western Blots}

Mice were perfused with phosphate-buffered saline, brains were isolated and different regions were dissected and homogenized in PBS supplemented with Complete protease inhibitor mixture (Roche) [12]. Protein concentrations were determined using the DC kit (Bio-Rad Laboratories). The homogenates were resolved on SDS-PAGE gels, transferred onto a polyvinylidene difluoride (PVDF) membrane, and hybridized with the 
antibodies raised against NDUFB8, NDUFA9, SDHA, UQCRFS1/RISP, VDAC1/Porin, CytC, COX1, ATP5a, UQCRC1/Core1, p62 (Abcam); GFAP, Synaptophysin, Nicastrin, Presenilin 1, LC3B (Cell Signaling); vinculin (Sigma); TUJ1 (Chemicon); Iba1 1:500 (Wako-016-20001); SOD2 1:2000 (Upstate); APP C-Terminal Fragment (Covance); 20s proteasome (Novus Biol). Depending on the size of the related protein, bands were normalized for housekeeping gene (vinculin) or for total protein loading (visualized by stain free technology, in the Chemidoc system, Biorad).

\section{Enzymatic Activity Assays}

Complex IV and citrate synthase activities were measured in tissue homogenates by spectrophotometric methods (41). Briefly, homogenates from cortex and hippocampus were prepared using a hand-held rotor (VWR) to homogenize tissue in PBS plus protease inhibitor mixture (Roche) on ice. Cytochrome $c(2 \mathrm{mM})$ reduced with sodium dithionite was added to homogenates in $10 \mathrm{mM} \mathrm{KH}_{2} \mathrm{PO}_{4}, 1 \mathrm{mg} / \mathrm{ml} \mathrm{BSA}$, and $120 \mathrm{mM}$ lauryl maltoside. Samples were read at $550-580 \mathrm{~nm}$ with the slope reading taken for $2^{\prime}$ at $37^{\circ} \mathrm{C}$. Potassium cyanide $(240 \mathrm{mM})$ was used to inhibit the reaction to ensure slope was specific to COX activity. Readings were normalized by protein concentration. Homogenates for citrate synthase were added to $50 \mathrm{mM}$ Tris- $\mathrm{HCl}, \mathrm{pH}$ 7.5, $20 \mathrm{mM}$ acetyl CoA, 10 mM 5,5'-dithiobis (2-nitrobenzoic acid), and 0.2\% Triton X-100 and performed at $412 \mathrm{~nm}$ with $50 \mathrm{mM}$ oxaloacetate to start the reaction. Readings were obtained for $5^{\prime}$ at $30^{\circ} \mathrm{C}$. Normalization was again to protein concentration.

\section{Beta-Secretase Activity Assay}

Beta-secretase activity was measured in cortical and hippocampal lysates using a FRET-based substrate, $\mathrm{H}$ RE(EDANS)EVNLDAEFK(DABCYL)R-OH (Calbiochem), which contains a beta-secretase cleavage sequence with Swedish-type mutations. Homogenates lysed in PBS- 1\% Triton-X and protease inhibitor cocktail (Roche) were centrifuged at $10,000 \times \mathrm{g}$ for $5^{\prime}$ at $4^{\circ} \mathrm{C}$, and the supernatant was used for the analysis. The activity assay was carried out on $4 \mu \mathrm{g}$ of protein in a $100-\mu$ l volume reaction volume that contained $12.5 \mu \mathrm{M}$ substrate and 20 $\mathrm{mM}$ sodium acetate $(\mathrm{pH} 4.4)$. The fluorescence from the released EDANS fluorophore was measured at $37^{\circ} \mathrm{C}$ with excitation and emission wavelengths of 355 and $460 \mathrm{~nm}$, respectively using a Synergy $\mathrm{H} 1$ hybrid reader (Biotek). Background fluorescence from the substrate alone was subtracted from the readings. Fluorescence (arbitrary units) was recorded 30' after the initiation of reaction.

\section{Quantification of $A \beta 42$ fragment}


Cortices and hippocampi were homogenized in cold guanidine buffer $[5 \mathrm{M}$ guanidine $\mathrm{HCl}, 50 \mathrm{mM}$ Tris- $\mathrm{HCl}(\mathrm{pH}$ 8.0)]. Total $A \beta 42$ content was quantified using a human $A \beta 42$ colorimetric ELISA kit (Invitrogen) according to the manufacturer's instruction with an incubation time of $16 \mathrm{~h}$ at $4^{\circ} \mathrm{C}$.

\section{Immunostaining and Stereological Quantification of Amyloid Plaques}

Anesthetized mice were perfused with ice-cold PBS, and 4\% PFA. Coronal sections were cut at a 20- $\mu$ m thickness by a cryostat from the rostral to caudal direction starting approximately at bregma -1.46 .

Sections were serially incubated in $70 \%$ formic acid for 20 ' and $0.3 \% \mathrm{H}_{2} \mathrm{O}_{2}$ in $100 \%$ methanol for 30 ', blocked with $10 \%$ normal goat serum (NGS) for 30' at RT. After blocking, sections were incubated with monoclonal anti-A $\beta$ $6 \mathrm{E} 10$ antibody (1:500; Chemicon) for $16 \mathrm{~h}$ at $4^{\circ} \mathrm{C}$. Slides were then incubated with secondary Antibody biotinconjugated goat anti-mouse (KPL) for $1 \mathrm{~h}$ at RT and Streptavidin-Peroxidase (KPL) for 30' at RT. Staining was visualized using a solution of $0.05 \%$ 3,3'-diaminobenzidine (DAB), $50 \mathrm{mM}$ Tris- $\mathrm{HCl} \mathrm{pH} 7.2,0.02 \% \mathrm{H}_{2} \mathrm{O}_{2}$. Slides were then mounted with SurgiPath Sub-X mounting media (Leica). Images were captured with an Pathscan Enabler 5. As previously described (35) the total number of amyloid plaques per tissue area (cerebral cortex and hippocampus) were counted from ten non-consecutive stained sections per animal ( $n=3-4$ per group) scanning the whole area. Fiji program was used for stereological counting.

Antibodies used for morphology analysis were: monoclonal anti-NeuN (1:500 Millipore), monoclonal anti-GFAP (1:500 Cell Signaling).

Nissl staining:

PFA-fixed frozen sections were dried for 3 hours, then incubated in 1:1 ethanol/chloroform overnight for defattening. Slides were then rehydrated through $100 \%$ ethanol to distilled water. Staining was performed in warm $\left(37^{\circ} \mathrm{C}\right) 0.1 \%$ cresyl violet solution for 5-10 minutes (Abcam 246817 ). Slides were then rinsed in running distilled water, serially dehydrated to $100 \%$ ethanol $2 \times 5$ minutes, cleared in xylene $2 \times 5$ minutes, and mounted with a permanent mounting medium. Images were captured with an Pathscan Enabler 5.

Immunostaining of Oxidized Nucleic Acids

Histological sections were obtained as previously described above. After deparaffinization and hydration, sections were microwave-heated twice for $4^{\prime}$ in $10 \mathrm{mM}$ sodium citrate buffer $(\mathrm{pH} 6.0)$. Sections were then permeabilized in $0.2 \%$ Triton $\mathrm{X}-100$ for 30 ' and endogenous peroxidase activity was quenched by immersing section in $100 \%$ methanol containing $0.3 \% \mathrm{H}_{2} \mathrm{O}_{2}$ for 30 '. After blocking sections with $10 \% \mathrm{NGS}$ for $1 \mathrm{~h}$ at $\mathrm{RT}$, sections were 
incubated with monoclonal antibody against 8-hydroxy-2'-deoxyguanosine (oh ${ }^{8} \mathrm{dG}$ ) and 8-hydroxyguanosine $\left(\right.$ oh $\left.^{8} \mathrm{G}\right)\left(1: 1,000\right.$; QED Bioscience) for $16 \mathrm{~h}$ at $4^{\circ} \mathrm{C}$ in $5 \%$ NGS. The bound primary antibody was detected as stated above using streptavidin peroxidase/DAB staining. For a negative control, AD sections were pre-incubated with $10 \mu \mathrm{g} / \mu \mathrm{l}$ RNase (Qiagen) and 0.04 units/ $\mu \mathrm{l}$ DNase (Promega) in $50 \mu \mathrm{l}$ of $1^{\prime} \mathrm{RQ}$ buffer (Promega) for 3 hours at $37^{\circ} \mathrm{C}$ prior to the primary antibody incubation. For a positive control, AD sections were pretreated with $30 \%$ $\mathrm{H}_{2} \mathrm{O}_{2}$ for 10 minutes before blocking with NGS.

OxyBlot

Cortices and hippocampi were homogenized in cold PBS + protein and phosphatase inhibitors. OxyBlot measures carbonyl groups introduced into proteins by oxidative reactions with ozone or oxides of nitrogen or by metal catalyzed oxidation. Carbonyl groups were quantified on $15 \mathrm{mg}$ of protein homogenates using S7150 SigmaAldrich OxyBlot Protein Oxidation Detection Kit.

\section{Statistical Analysis}

Two-tailed, unpaired Student $t$ test was used to determine the statistical significance between the different groups. If more than two groups were analyzed, significance of the differences was evaluated by one-way ANOVA followed by Bonferroni post-test. Double asterisks indicate $p<0.01$, a single asterisk indicates $p<0.05$. Error bars represent standard error of the mean (SEM).

\section{Results}

\section{Generation of $\mathrm{CIII}^{\mathrm{KO}}-\mathrm{AD}$ mice}

To analyze the effects of Complex III depletion on $A D$ pathology, we developed and characterized an $A D$ transgenic mouse carrying mutant APP and mutant presenilin 1 (APP/PS1) (37) with adult-onset Rieske Iron Sulfur Protein (RISP) defects. RISP is encoded by the UQCRFS1 gene and is a catalytic subunit of mitochondrial OXPHOS Complex III. Because APP/PS1 mice develop amyloid deposits in cortex and hippocampus starting at 6-7 months of age (42), we wanted to induce a Complex III defect in adult mice. Therefore, we used a CaMKIlaCreERT2 transgenic mouse in which the expression of the Cre recombinase is tamoxifen-inducible (38) (Fig.1B). The experimental animals' (CIII $\left.{ }^{\mathrm{KO}}-\mathrm{AD}\right)$ genotype was RISP ${ }^{\mathrm{F} / \mathrm{F}}-\mathrm{APP} / \mathrm{PS} 1^{+/-}-\mathrm{CaMKIla}-\mathrm{CreERT}{ }^{+/-}$, whereas the control animals' (AD) genotype was RISP/F-APP/PS1 ${ }^{+/-}$(Fig.1A). CaMKIla is expressed also in male germ cells (43), so in order to obtain the experimental animals, only females CaMKIla-CreERT2 ${ }^{+/-}$were used in the breeding 
scheme (Fig.1A). We injected 5-month-old mice with tamoxifen, and we analyzed the AD pathology at 8 months, 3 months after inducing the UQCRFS1 gene deletion.

To verify that cre recombinase excised exon 2 of the UQCRFS1 gene specifically in brain of CIII ${ }^{\mathrm{KO}}-\mathrm{AD}$ mice after tamoxifen induction, we analyzed DNA extracted from cortex and spleen of CIII ${ }^{\mathrm{KO}}-\mathrm{AD}$ and $\mathrm{AD}$ mice injected with tamoxifen (Fig.1B). We could detect the gene deletion in cortex of $\mathrm{CIII}^{\mathrm{KO}}-\mathrm{AD}$ mice, but not in spleen of $\mathrm{CIII}^{\mathrm{KO}}-\mathrm{AD}$ mice and in cortex of control mice (Fig1C), indicating the specificity of the system. We estimated the levels of exon 2 excision in cortex and hippocampus by qPCR and found that both areas had 50\% of the UQCRFS1 gene deleted (Fig.1D). Considering that the samples used to extract DNA contained not only CaMKIlla neurons but also glia and CaMKIla- neurons, the excision of the UQCRFS1 gene in the target neurons was actually higher than the $50 \%$ detected by this method. No significant differences were detected in the excision levels between cortex and hippocampus (Fig.1E).

\section{Effects of ablation of RISP on OXPHOS complexes}

To verify the levels of RISP protein in CaMKIIa+ neurons, we performed a western blot assay on cortical and hippocampal homogenates of $A D$ and CIIIKO-AD mice (Fig.2). When normalized to total protein loading, RISP protein was not reduced in cortex (Fig. 2A-2D) or in hippocampus (Fig. 2A-2G). When normalized to a mitochondrial membrane protein, VDAC, the level of RISP was reduced to $62 \%$ in cortex (Fig.2B) and to $77 \%$ in hippocampus (Fig.2E) compared to controls (females). We also normalized RISP to steady-state level of UQCRC1 (Core 1, another Complex III subunit located in a different module of the fully assembled Complex III) and calculated that RISP was reduced to about $71 \%$ in cortex (Fig.2C) and to $88 \%$ in hippocampus (Fig.2F) compared to controls. To determine whether the decrease of the Complex III subunits affected the other OXPHOS complexes, we analyzed the steady-state levels of representative proteins from each complex. We analyzed NDUFS3 and NDUFB8 (for Complex I), SDHA (for Complex II), COXI and CYTC (for Complex IV). We detected a significant increase of Complex II subunit (SDHA) in both cortex and hippocampus but no other proteins belonging to the other complexes were significantly affected. We performed this analysis also in males (Suppl. Fig.1) which also showed an increase of SDHA in the hippocampal homogenates. Interestingly, in males, COXI (Complex IV) was also significantly increased in the CIIIKO-AD mice compared to control. To determine if the increased levels of subunits of Complex II in females and of Complex II and IV in males were due to increased 
mitochondrial mass, we measured the steady-state levels of two other non-OXPHOS mitochondrial membrane proteins, VDAC1 and TIM23. VDAC1 was increased in cortex from both females and males but unchanged in hippocampus (Fig. 2H-I, Suppl.Fig.1H-I). TIM23 was increased only in hippocampal homogenates from females (Fig. 2H-I).

To determine if the induced decrease of RISP was enough to cause a defect in Complex III activity, we measured OXPHOS complex enzymatic activities in cortical and hippocampal homogenates. Complex III activity was reduced in cortex to $65 \%( \pm 7)$ compared to control (Fig.3A), in hippocampus it was reduced to $61 \%( \pm 3.5)$ (Fig.3C). These homogenates also contain glial and endothelial cells, in which the UQCRFS1 gene has not been knocked out. Because of this, we have to consider an underestimation of the actual decrease in Complex III activity in affected neurons. Complex IV and citrate synthase (CS) activity were not changed in cortical and hippocampal homogenates (Fig.3B, 3D, Suppl. Fig.2).

In order to analyze if the loss of RISP caused a reorganization of the respiratory complexes, mitochondrial proteins were extracted with digitonin and separated by blue native gel electrophoresis followed by western blot. Complex I, III and IV were analyzed using anti-NDUFA9, RISP and COX1 antibodies, respectively. TIM23, VDAC1 and SDHA were used as mitochondrial loading control. Mitochondrial supercomplexes are indicated in the figure as HMW (High Molecular Weight) and $\mathrm{Cl}+\mathrm{CIII}_{2}$. Complex V and II were analyzed using ATP5a and SDHA antibodies, respectively. We detected a decrease of $\mathrm{Cl}+\mathrm{CIII}_{2}$ architectures in cortex of $\mathrm{CIII}{ }^{\mathrm{KO}}-\mathrm{AD}$ female mice, and an increase of free Complex I (Fig.3E-F). We found similar results in hippocampus, but because of a higher variability, the analysis did not reach statistical significance (Fig.3E-G). Assembled Complexes IV, V and II did not show changes.

\section{Neurodegeneration and neuroinflammation in CIII ${ }^{\mathrm{KO}}-\mathrm{AD}$ mice}

The brains of 8-month-old CIIIKO-AD mice did not show significant differences in size or weight compared to AD controls. To detect if the mild defect in Complex III affected the neuroanatomy of the brain, we first analyzed the gross morphology of different brain regions by hematoxylin \& eosin staining. We did not detect any gross nor massive anatomical alteration (Suppl. Fig. 3A). We then performed Nissl staining (which is commonly use to stain nucleic acids in the nervous tissue) and immunohistochemistry staining for NeuN (marker of neuronal nuclei) on PFA-fixed frozen sections. We did not detect gross changes in the neuronal population in different areas 
analyzed (Suppl. Fig. 3B, Fig.4A). We then performed western blots analysis to measure the levels of neuronal marker TUJ1 and synaptic marker Synaptophysin on cortical and hippocampal homogenates of CIIIKO-AD and AD controls. We detected a decrease in TUJ1 in cortex from CIII ${ }^{\mathrm{KO}}-\mathrm{AD}$ females compared to AD controls (Fig.4B), while we did not detect significant changes in males (Suppl. Fig.3D).

Neurodegeneration and metabolic defects are often accompanied by neuroinflammation, therefore we examined the extent of gliosis in these animals. We performed immunohistochemistry and western blot analysis with antibodies against GFAP (marker of glial cells) and Iba1 (marker of microglia) on both females and males. We did not detect significant changes, neither in the steady-state levels of both markers (Fig. 4D, Suppl. Fig.3F) nor in the morphology of GFAP and Iba1 positive cells (Fig.4C, Suppl. Fig.3E).

\section{CIII ${ }^{\mathrm{KO}}-\mathrm{AD}$ mice show fewer amyloid plaques and fewer $\mathrm{A} \beta 42$ fragment}

Starting at 6 months of age, $A D$ mice accumulate numerous amyloid plaques, with most of them present in the cerebral cortex and the hippocampus (36). By 8-9 months, the plaque number reaches 1.5-2 plaques per $\mathrm{mm}^{2}$ $(35,36)$. To analyze if the partial defect of Complex III affected the formation of the amyloid plaques, we performed a stereological count of amyloid plaques on 8-month-old $A D$ and CIII ${ }^{K O}-A D$ mice. We visualized the plaques by immunohistochemistry on serial coronal sections with an anti-human $A \beta$ antibody (6E10) that recognizes the amyloidogenic portion of APP. Brains from CIII ${ }_{-}$AD females showed significant reductions in the number of plaques compared to $A D$ brains, in cortex and hippocampus (Fig. 5A-B).

The plaque number is correlated with $A \beta 42$ content and to secretase activity $(44,45)$. To determine whether the diminished number of plaques in CIIIKO-AD mice correlated with the amount of $A \beta 42$ peptides, we performed an ELISA on protein homogenates from cortex and hippocampus of $A D$ and CIII ${ }_{-} A D$ mice. Lysates from CIII ${ }_{-} A D$ females' brains showed a reduction of $\mathrm{A} \beta 42$ of $\sim 66 \%$ in cortex and of $\sim 50 \%$ in hippocampus (Fig.5C). We consequently analyzed APP processing by performing western blot analyses of the different fragments derived from the APP sequential cleavage. We used an antibody against the carboxy terminal portion of APP (Ctf, amino acid residues 676-695) that recognizes both the un-cleaved APP and the C-terminal fragment, formed after the first secretase cleavage (either by alpha- or beta-secretase). From this analysis we detected a decrease in the ratio between the carboxy terminal fragment (Ctf) and the full-length APP in homogenates from the cortex, suggesting a downregulation of this processing (Fig. 5D). In hippocampus we did not detect significant changes. 
One of the crucial events triggering the formation of amyloid fragments from APP is the cleavage by betasecretase. We measured beta-secretase activity with a FRET-based assay in cortical lysates from AD and CIIIKO_ AD mice brains, but we did not detect significant differences between the 2 groups (Fig. 5E), suggesting that a decrease in beta-secretase activity was not responsible for the reduced levels of Ctf/APP. The steady state levels of the major gamma-secretases involved in the formation of $A \beta 42$ (Nicastrin and Presenilin 1) in cortical and hippocampal homogenates, were also not altered (Fig.5F). To investigate the possibility that the decrease in $A \beta 42$ fragments occurred because of an increase in its degradation, we analyzed the levels of two proteins involved in general cellular pathways for protein degradation: p62 and LC3B (autophagy pathway), and 20S proteasome (ubiquitin proteasome system, UPS). We did not detect significant changes in the levels of p62 nor in LC3B lipidation (Suppl. Fig.4A). We did detect a decrease of the $20 \mathrm{~S}$ proteasome levels in cortical but not in hippocampal homogenates (Suppl. Fig.4B). Brains from CIIIKO-AD mice males, did not show significant changes in plaque numbers (Suppl. Fig.5A-B), Aß42 content (Suppl. Fig.5C), or APP processing (Suppl. Fig.5D).

\section{CIII ${ }^{\mathrm{KO}}-\mathrm{AD}$ mice show increased oxidative stress}

Plaque formation has been correlated with oxidative stress. AD patient brains show increased levels of lipid peroxidation, DNA strand breaks, and oxidized DNA bases (46). To investigate for signs of oxidative stress, we measured the steady-state level of SOD2, a protein that is typically increased as a consequence of oxidative stress. We detected increased levels of SOD2 in cortex of CIIIKO-AD females compared to AD, while in hippocampus we did not detect changes (Fig.6A). Oxidative stress also causes oxidative modification to proteins with consequent addition of carbonyl groups to protein side chains. To provide a more direct indication of the oxidative state of the different brain regions, we performed an OxyBlot (Sigma), that identifies these carbonyl groups. Cortical homogenates showed proteins with more carbonylated groups, indicating a higher status of oxidative stress (Fig. 6B). Hippocampus did not show any significant changes, similar to the analysis performed with SOD2.

Previously we showed that the cRISPKO animals displayed oxidative stress in specific regions of the brain, in particular in the piriform cortex (47). To analyze if in our model the oxidative stress was also localized in specific regions of the brain, we performed an 8-hydroxy-deoxy-guanosine (8-OHdG)/8-hydroxy-guanosine (8-OHG) staining. 8-OHdG and 8-OHG are a product of DNA and RNA oxidative damage respectively, therefore an indirect 
result of the presence of ROS. We did not detect changes between $A D$ and CIIIKO-AD mice (Fig. 6C), showing that the mild CIII defect did not exacerbate oxidative damage to nucleic acids.

\section{Discussion}

Although the primary cause of Alzheimer's disease (AD) is still unknown, mitochondrial OXPHOS dysfunction has been implicated in the development and progression of AD. Several studies reported OXPHOS defects in postmortem $\operatorname{AD}$ brains $(14,16,17,48)$ and mutations in mitochondrial DNA (mtDNA), which encodes proteins for several OXPHOS complexes (10-12). Moreover, it has been shown recently that mitochondrial Complex I abnormalities are associated with tau load in AD patients (49). By better understanding the metabolic consequences of OXPHOS defects in the CNS and in AD pathology, novel therapeutic approaches potentially applicable to $A D$ could be developed.

Different OXPHOS deficiencies influence development and brain pathology in distinctive ways. We have previously compared the phenotypes of mice in which Complex I (NDUFS3) (50), Complex III (RISP) or Complex IV (COX10) (47) have been knocked out in neuronal cells. These models showed some interesting differences. The Complex I neuronal KO (Ndufs $3 \mathrm{nKO}$ ) mice died around 5 months with a fatal encephalopathy, ataxia, neuronal cell death and massive gliosis (50). The Complex III deficient mice (Uqcrfs1 nKO) were all dead by 5 months and showed degeneration of the piriform cortex with high levels of oxidative damage (47). The Complex IV deficient mice (Cox10 nKO) survived much longer with a progressive CNS defect and showed a neuronal loss localized to the hippocampus (47). These findings are also different from our observations with a model of neuronal mtDNA damage, which showed prominent degeneration of the striatum prior to the degeneration of the cortex and hippocampus (51).

Therefore, different OXPHOS deficiencies could influence the development of AD pathology in distinctive ways. Focusing on the amyloid cascade, it is surprising that in most of the studies on mouse models of $A D$, inhibition of different mitochondrial complexes resulted in a decrease of plaque burden and $A \beta$ accumulation. Zhang et al. (52) reported how a mild inhibition of Complex I reduced levels of $A \beta$ and phospho-Tau in three AD animal models. In these animals, there was no sign of oxidative damage or inflammation and mitochondrial bioenergetics was increased. AMP-activated protein kinase was upregulated, GSK3 $\beta$ activity was reduced, and restoration of axonal trafficking resulted in elevated levels of neurotrophic factors and synaptic proteins (52). Our lab previously 
generated a neuron-specific Complex IV conditional KO mouse (knocking out Cox10, subunit of CIV) crossed with the APP/PS1 mouse (COXd-AD) (35). The plaque burden in cortex and hippocampus was significantly decreased in COXd-AD mice compared to AD controls. This reduction was accompanied by a reduction of total A 342 without a significant alteration in the level of APP. In that model, beta-secretase activity was lower and oxidative stress was reduced. The $A \beta$ pathology in this model, however, was studied at 4 months, because the ablation of COX10 caused a massive neurodegeneration.

In a different model (AD-mito-Pstl), we induced mtDNA damage in cortex and hippocampus of APP/PS1 mouse for 2 months ( 6 to 8 months of age) (36). Also, in this model, the OXPHOS deficiency caused by mtDNA depletion had a negative effect on $A \beta 42$ content and, consequently, on plaque burden. This effect was associated with an alteration in APP processing, independent from beta-secretase activity and from oxidative stress. On the other hand, in the mutator mouse, that show accumulation of mtDNA mutations and premature aging, $A \beta 42$ levels and plaque density were increased. The increased amyloid pathology was not caused by an elevated $A \beta$ production but by a decreased clearance by the insulin degrading enzyme (IDE) (53).

A frequent criticism, leveled by proponents of OXPHOS defects causing plaque and $A \beta$ accumulation, is that the OXPHOS defect in these different models was too severe and already present from birth, not mimicking mild defects associated with normal aging. To address this criticism, we analyzed the consequences of a mild and adult-onset Complex III deficiency on the amyloid pathology. We chose Complex III, because defects in this complex have been associated with increased ROS (47). The lack of Complex III activity in neurons (analyzed in previous RISP nKO mice) caused premature death by 5 months of age. To avoid such a strong phenotype, we used an inducible model in which the expression of cre-recombinase is activated by tamoxifen injections at 5months of age. This caused a deletion of encoded protein Rieske iron-sulfur protein (RISP), catalytic subunit of Complex III, with a consequent mild Complex III deficiency in CamKIla positive neurons. This mild CIII deficiency allowed us to avoid a massive neurodegeneration and to analyze the amyloid pathology 3 months later, at 8 months of age.

There was a difference in phenotypes between females and males. Sex differences in APP/PS1 mice are well known, with females accumulating amyloid at an earlier age than males and building up more amyloid deposits (54). These differences are not limited to the AD pathology (55-58). In our model, the decrease in plaque burden and $A \beta$ fragment was evident only in cortex and hippocampus of female mice, while amyloid pathology in males 
was not affected by the defect of Complex III. Moreover, we detected a mild decrease of TUJ1 in cortex of females but no change in the same regions in males.

Still, this model also showed a decrease of plaque burden caused by a lower amount of A $\beta 42$ fragment. In cortex, this was accompanied by an alteration in the APP processing (lower level of Ctf/APP), but not by a difference in secretases activity or steady-state levels. Oxidative stress might affect the plaque formation by enhancing the expression and activity of BACE-1 $(59,60)$ and by enhanced secretion of soluble APP $\beta$. In vitro and in vivo studies showed how Complex I and III- derived ROS lead to elevated levels of A (24). Moreover, recent studies have also reported that the treatment of different $A D$ mouse models with mitochondrial-targeted antioxidants have protective effects that includes decrease in $A \beta$ production and plaque accumulation $(61,62)$. Therefore, the working hypothesis in the previous models was that a lower level of oxidative stress might have been the cause of a decrease in plaque number (35).

The fact that in our model we detected increased oxidative stress, led us to the conclusion that the mechanism by which interfering with OXPHOS complexes activity leads to a decrease of plaques is independent from ROS formation. The consideration that in the models in which mtDNA and Complex I were impaired, the decreased number of plaques was accompanied by no change in ROS, also support our conclusion. Although ROS can exacerbate $A D$ pathology, it is not clear if it has a primary role in the development of the disease.

What may be more compatible with the existing data, is that mitochondria dysfunction is a consequence of $A \beta$ toxicity. The $A \beta$ fragment's typical localization is extracellular, but different studies showed that it can be found in other organelles, including mitochondria, in both human AD brain and mouse models of $A D$ where it causes impairment of electron transport chain (ETC) complexes and increased ROS production $(32,63)$. A $\beta$ can actively be imported into mitochondria through cellular trafficking systems and colocalize with the ETC inhibiting Complex IV $(29,64)$. A $\beta$ may also contribute to mitochondrial dysfunction through disruption of mitochondrial fusion and fission processes, leading to mitochondrial fragmentation (34).

Our results, together with previous reports, support a model in which mitochondrial dysfunction in AD mouse models is not the cause of $A \beta$ oligomer accumulation. Rather, our data are more compatible with a model in which OXPHOS function is decreased because of $A \beta$ toxicity. 


\section{Conclusions}

We reported how mitochondrial Complex III dysfunction in adult neurons of a mouse model of amyloid pathology induced mild oxidative stress which did not correlate with increased amyloid beta accumulation. CIII ${ }^{\mathrm{KO}}-\mathrm{AD}$ mice showed decreased plaque number, decreased $A \beta 42$ toxic fragment and altered amyloid precursor protein cleavage pathway. Our results support a model in which mitochondrial dysfunction is not the cause of amyloid pathology but rather a consequence of $A \beta$ toxicity.

\section{List of abbreviations:}

A $\beta$ : Amyloid beta

AD: Alzheimer's disease

APP/PS1: amyloid precursor protein (Mo/HuAPP695swe), presenilin 1 (PS1-dE9).

ATP5a: ATP synthase alpha-subunit gene

BN-PAGE: Blue native polyacrylamide gel electrophoresis

CaMKIla: calcium/calmodulin-dependent protein kinase II alpha

COX1: Cyclooxygenase 1 (Cytochrome c oxidase)

CS: Citrate Synthase

CTF: APP C-Terminal Fragment

CytC: Cytochrome C

GFAP: Glial fibrillary acidic protein

Iba1: Ionized calcium binding adaptor molecule 1

LC3B: Autophagy marker Light Chain 3

mtDNA: mitochondrial DNA

NDUFB8: NADH:Ubiquinone Oxidoreductase Subunit B8

NDUFA9: NADH:Ubiquinone Oxidoreductase Subunit A9

OXPHOS: Oxidative Phosphorylation

PBS: Phosphate-buffered saline

PFA: Paraformaldehyde

PGC-1a: peroxisome proliferator-activated receptor gamma coactivator 1-alpha; 
PVDF: polyvinylidene difluoride

RISP: Rieske Iron Sulfur Protein

ROS: reactive oxygen species

$\mathrm{RT}$ : room temperature

SDHA, Succinate Dehydrogenase Complex Flavoprotein Subunit A

SDS-PAGE: sodium dodecyl sulfate polyacrylamide gel electrophoresis

SOD2: Superoxide dismutase 2

TUJ1: Neuron-specific class III beta-tubulin

UQCRC1/Core1: Ubiquinol-Cytochrome C Reductase Core Protein 1

UQCRFS1: Ubiquinol-Cytochrome C Reductase, Rieske Iron-Sulfur Polypeptide 1

VDAC1/Porin: voltage-dependent anion channel

\section{Declarations:}

\section{Ethical Approval and Consent to participate}

Not applicable

\section{Consent for publication}

All authors consented to the publication. Francisca Diaz passed away prior to the submission of the research paper.

\section{Availability of supporting data}

The datasets used and/or analyzed during the current study are available from the corresponding author on reasonable request.

\section{Competing interests}

The authors declare no competing interests.

\section{Funding}

This work was supported primarily by the Florida Biomedical Foundation Ed and Ethel Moore Alzheimer's Disease Research Program grant 5AZ06 (CTM), the National Institute of Health Grants K01AG057815 (MP), and 1R01NS079965 (CTM).

Authors' contributions 
MP designed the research, performed the experiments, analyzed, and interpreted data, and wrote the manuscript. FD performed BN-PAGE analysis, enzymatic activity, and contributed intellectually to the research. NN performed qPCR analysis CSG and PI assisted in plaque counting, RB contributed intellectually to the research, CTM planned the project together with MP and contributed to the writing of the manuscript. All authors read and approved the final manuscript.

\section{Acknowledgements:}

We thank the University of Miami Comparative Pathology Laboratory. The following grants also helped support this work: NIH grants 5R01EY010804, 1R01AG036871, R33ES025673 and the Muscular Dystrophy Association.

\section{Authors' information:}

${ }^{1}$ Department of Neurology, University of Miami Miller School of Medicine. Neurology Research Building, 1420 NW 9th Avenue, Miami, FL 33136, USA.

${ }^{2}$ The Miami Project to Cure Paralysis, Department of Neurological Surgery, University of Miami Miller School of Medicine, Lois Pope Life Center, 1095 NW 14th Terrace, Miami, FL 33136.

Francisca Diaz passed away before the submission of the manuscript.

Correspondence: mpinto@med.miami.edu or cmoraes@med.miami.edu

\section{References}

1. Polanco JC, Li C, Bodea LG, Martinez-Marmol R, Meunier FA, Gotz J. Amyloid-beta and tau complexity towards improved biomarkers and targeted therapies. Nat Rev Neurol. 2018;14(1):22-39.

2. Swerdlow RH, Burns JM, Khan SM. The Alzheimer's disease mitochondrial cascade hypothesis: progress and perspectives. Biochim Biophys Acta. 2014;1842(8):1219-31.

3. Tapias V. Editorial: Mitochondrial Dysfunction and Neurodegeneration. Front Neurosci. 2019;13:1372.

4. Hou Y, Dan X, Babbar M, Wei Y, Hasselbalch SG, Croteau DL, et al. Ageing as a risk factor for neurodegenerative disease. Nat Rev Neurol. 2019;15(10):565-81.

5. Bowling AC, Mutisya EM, Walker LC, Price DL, Cork LC, Beal MF. Age-dependent impairment of mitochondrial function in primate brain. J Neurochem. 1993;60(5):1964-7.

6. Hauptmann S, Scherping I, Drose S, Brandt U, Schulz KL, Jendrach M, et al. Mitochondrial dysfunction: an early event in Alzheimer pathology accumulates with age in AD transgenic mice. Neurobiol Aging. 2009;30(10):1574-86.

7. Hong MG, Myers AJ, Magnusson PK, Prince JA. Transcriptome-wide assessment of human brain and lymphocyte senescence. PloS one. 2008;3(8):e3024. 
8. Ross JM, Oberg J, Brene S, Coppotelli G, Terzioglu M, Pernold K, et al. High brain lactate is a hallmark of aging and caused by a shift in the lactate dehydrogenase A/B ratio. Proc Natl Acad Sci U S A. 2010;107(46):20087-92.

9. Yao J, Irwin RW, Zhao L, Nilsen J, Hamilton RT, Brinton RD. Mitochondrial bioenergetic deficit precedes Alzheimer's pathology in female mouse model of Alzheimer's disease. Proc Natl Acad Sci U S A. 2009;106(34):14670-5.

10. Coskun PE, Beal MF, Wallace DC. Alzheimer's brains harbor somatic mtDNA control-region mutations that suppress mitochondrial transcription and replication. Proc Natl Acad Sci U S A. 2004;101(29):10726-31.

11. Krishnan KJ, Ratnaike TE, De Gruyter HL, Jaros E, Turnbull DM. Mitochondrial DNA deletions cause the biochemical defect observed in Alzheimer's disease. Neurobiol Aging. 2012;33(9):2210-4.

12. Lin MT, Simon DK, Ahn CH, Kim LM, Beal MF. High aggregate burden of somatic mtDNA point mutations in aging and Alzheimer's disease brain. Hum Mol Genet. 2002;11(2):133-45.

13. Bennett JP, Jr., Keeney PM. Alzheimer's and Parkinson's brain tissues have reduced expression of genes for mtDNA OXPHOS Proteins, mitobiogenesis regulator PGC-1alpha protein and mtRNA stabilizing protein LRPPRC (LRP130). Mitochondrion. 2020;53:154-7.

14. Chagnon P, Betard C, Robitaille Y, Cholette A, Gauvreau D. Distribution of brain cytochrome oxidase activity in various neurodegenerative diseases. Neuroreport. 1995;6(5):711-5.

15. Chen JX, Yan SS. Role of mitochondrial amyloid-beta in Alzheimer's disease. J Alzheimers Dis. 2010;20 Suppl 2:S569-78.

16. Long J, He P, Shen Y, Li R. New evidence of mitochondria dysfunction in the female Alzheimer's disease brain: deficiency of estrogen receptor-beta. J Alzheimers Dis. 2012;30(3):545-58.

17. Mutisya EM, Bowling AC, Beal MF. Cortical cytochrome oxidase activity is reduced in Alzheimer's disease. J Neurochem. 1994;63(6):2179-84.

18. Holper L, Ben-Shachar D, Mann JJ. Multivariate meta-analyses of mitochondrial complex I and IV in major depressive disorder, bipolar disorder, schizophrenia, Alzheimer disease, and Parkinson disease. Neuropsychopharmacology. 2019;44(5):837-49.

19. Onyango IG, Khan SM, Bennett JP, Jr. Mitochondria in the pathophysiology of Alzheimer's and Parkinson's diseases. Frontiers in bioscience. 2017;22:854-72.

20. Kim SH, Vlkolinsky R, Cairns N, Lubec G. Decreased levels of complex III core protein 1 and complex V beta chain in brains from patients with Alzheimer's disease and Down syndrome. Cell Mol Life Sci. 2000;57(12):1810-6.

21. Kenney PM, Bennett JP, Jr. Alzheimer's Disease Frontal Cortex Mitochondria Show a Loss of Individual Respiratory Proteins but Preservation of Respiratory Supercomplexes. Int J Alzheimers Dis.

2019;2019:4814783.

22. Fisar Z, Hansikova H, Krizova J, Jirak R, Kitzlerova E, Zverova M, et al. Activities of mitochondrial respiratory chain complexes in platelets of patients with Alzheimer's disease and depressive disorder. Mitochondrion. 2019;48:67-77.

23. Guglielmotto $M$, Aragno M, Autelli R, Giliberto L, Novo E, Colombatto $S$, et al. The up-regulation of BACE1 mediated by hypoxia and ischemic injury: role of oxidative stress and HIF1alpha. J Neurochem. 2009;108(4):1045-56.

24. Leuner K, Schutt T, Kurz C, Eckert SH, Schiller C, Occhipinti A, et al. Mitochondrion-derived reactive oxygen species lead to enhanced amyloid beta formation. Antioxid Redox Signal. 2012;16(12):1421-33.

25. Paola D, Domenicotti C, Nitti M, Vitali A, Borghi R, Cottalasso D, et al. Oxidative stress induces increase in intracellular amyloid beta-protein production and selective activation of betal and betall PKCs in NT2 cells. Biochem Biophys Res Commun. 2000;268(2):642-6.

26. Tamagno E, Bardini $P$, Obbili A, Vitali A, Borghi R, Zaccheo D, et al. Oxidative stress increases expression and activity of BACE in NT2 neurons. Neurobiol Dis. 2002;10(3):279-88. 
27. Anandatheerthavarada HK, Biswas G, Robin MA, Avadhani NG. Mitochondrial targeting and a novel transmembrane arrest of Alzheimer's amyloid precursor protein impairs mitochondrial function in neuronal cells. The Journal of cell biology. 2003;161(1):41-54.

28. Calkins MJ, Manczak M, Mao P, Shirendeb U, Reddy PH. Impaired mitochondrial biogenesis, defective axonal transport of mitochondria, abnormal mitochondrial dynamics and synaptic degeneration in a mouse model of Alzheimer's disease. Hum Mol Genet. 2011;20(23):4515-29.

29. Casley CS, Canevari L, Land JM, Clark JB, Sharpe MA. Beta-amyloid inhibits integrated mitochondrial respiration and key enzyme activities. J Neurochem. 2002;80(1):91-100.

30. Devi L, Prabhu BM, Galati DF, Avadhani NG, Anandatheerthavarada HK. Accumulation of amyloid precursor protein in the mitochondrial import channels of human Alzheimer's disease brain is associated with mitochondrial dysfunction. J Neurosci. 2006;26(35):9057-68.

31. Hansson Petersen CA, Alikhani N, Behbahani H, Wiehager B, Pavlov PF, Alafuzoff I, et al. The amyloid beta-peptide is imported into mitochondria via the TOM import machinery and localized to mitochondrial cristae. Proc Natl Acad Sci U S A. 2008;105(35):13145-50.

32. Manczak M, Anekonda TS, Henson E, Park BS, Quinn J, Reddy PH. Mitochondria are a direct site of A beta accumulation in Alzheimer's disease neurons: implications for free radical generation and oxidative damage in disease progression. Hum Mol Genet. 2006;15(9):1437-49.

33. Reddy PH, Beal MF. Amyloid beta, mitochondrial dysfunction and synaptic damage: implications for cognitive decline in aging and Alzheimer's disease. Trends in molecular medicine. 2008;14(2):45-53.

34. Wang X, Su B, Siedlak SL, Moreira PI, Fujioka H, Wang Y, et al. Amyloid-beta overproduction causes abnormal mitochondrial dynamics via differential modulation of mitochondrial fission/fusion proteins. Proc Natl Acad Sci U S A. 2008;105(49):19318-23.

35. Fukui H, Diaz F, Garcia S, Moraes CT. Cytochrome c oxidase deficiency in neurons decreases both oxidative stress and amyloid formation in a mouse model of Alzheimer's disease. Proc Natl Acad Sci U S A. 2007;104(35):14163-8.

36. Pinto M, Pickrell AM, Fukui $H$, Moraes CT. Mitochondrial DNA damage in a mouse model of Alzheimer's disease decreases amyloid beta plaque formation. Neurobiol Aging. 2013;34(10):2399-407.

37. Jankowsky JL, Slunt HH, Ratovitski T, Jenkins NA, Copeland NG, Borchelt DR. Co-expression of multiple transgenes in mouse CNS: a comparison of strategies. Biomol Eng. 2001;17(6):157-65.

38. Madisen L, Zwingman TA, Sunkin SM, Oh SW, Zariwala HA, Gu H, et al. A robust and high-throughput Cre reporting and characterization system for the whole mouse brain. Nat Neurosci. 2010;13(1):133-40.

39. Waypa GB, Marks JD, Guzy RD, Mungai PT, Schriewer JM, Dokic D, et al. Superoxide generated at mitochondrial complex III triggers acute responses to hypoxia in the pulmonary circulation. Am J Respir Crit Care Med. 2013;187(4):424-32.

40. Schmittgen TD, Livak KJ. Analyzing real-time PCR data by the comparative $\mathrm{C}(\mathrm{T})$ method. Nat Protoc. 2008;3(6):1101-8.

41. Barrientos A, Fontanesi F, Diaz F. Evaluation of the mitochondrial respiratory chain and oxidative phosphorylation system using polarography and spectrophotometric enzyme assays. Curr Protoc Hum Genet. 2009; Chapter 19:Unit19 3.

42. Jankowsky JL, Fadale DJ, Anderson J, Xu GM, Gonzales V, Jenkins NA, et al. Mutant presenilins specifically elevate the levels of the 42 residue beta-amyloid peptide in vivo: evidence for augmentation of a 42-specific gamma secretase. Hum Mol Genet. 2004;13(2):159-70.

43. Choi Cl, Yoon SP, Choi JM, Kim SS, Lee YD, Birnbaumer L, et al. Simultaneous deletion of floxed genes mediated by CaMKIlalpha-Cre in the brain and in male germ cells: application to conditional and conventional disruption of Goalpha. Exp Mol Med. 2014;46:e93.

44. Mohajeri MH, Saini KD, Nitsch RM. Transgenic BACE expression in mouse neurons accelerates amyloid plaque pathology. J Neural Transm (Vienna). 2004;111(3):413-25. 
45. Li R, Lindholm K, Yang LB, Yue X, Citron M, Yan R, et al. Amyloid beta peptide load is correlated with increased beta-secretase activity in sporadic Alzheimer's disease patients. Proc Natl Acad Sci U S A. 2004;101(10):3632-7.

46. Misrani A, Tabassum S, Yang L. Mitochondrial Dysfunction and Oxidative Stress in Alzheimer's Disease. Frontiers in aging neuroscience. 2021;13:617588.

47. Diaz F, Garcia S, Padgett KR, Moraes CT. A defect in the mitochondrial complex III, but not complex IV, triggers early ROS-dependent damage in defined brain regions. Hum Mol Genet. 2012;21(23):5066-77.

48. Sheng B, Wang X, Su B, Lee HG, Casadesus G, Perry G, et al. Impaired mitochondrial biogenesis contributes to mitochondrial dysfunction in Alzheimer's disease. J Neurochem. 2012;120(3):419-29.

49. Terada T, Therriault J, Kang MSP, Savard M, Pascoal TA, Lussier F, et al. Mitochondrial complex I abnormalities is associated with tau and clinical symptoms in mild Alzheimer's disease. Molecular Neurodegeneration. 2021;16(1):28.

50. Peralta S, Pinto M, Arguello T, Garcia S, Diaz F, Moraes CT. Metformin delays neurological symptom onset in a mouse model of neuronal complex I deficiency. JCI Insight. 2020;5(21).

51. Pickrell AM, Fukui $\mathrm{H}$, Wang $\mathrm{X}$, Pinto $\mathrm{M}$, Moraes $\mathrm{CT}$. The striatum is highly susceptible to mitochondrial oxidative phosphorylation dysfunctions. J Neurosci. 2011;31(27):9895-904.

52. Zhang L, Zhang S, Maezawa I, Trushin S, Minhas P, Pinto M, et al. Modulation of mitochondrial complex I activity averts cognitive decline in multiple animal models of familial Alzheimer's Disease. EBioMedicine. 2015;2(4):294-305.

53. Kukreja L, Kujoth GC, Prolla TA, Van Leuven F, Vassar R. Increased mtDNA mutations with aging promotes amyloid accumulation and brain atrophy in the APP/Ld transgenic mouse model of Alzheimer's disease. Mol Neurodegener. 2014;9:16.

54. Wang J, Tanila H, Puolivali J, Kadish I, van Groen T. Gender differences in the amount and deposition of amyloidbeta in APPswe and PS1 double transgenic mice. Neurobiol Dis. 2003;14(3):318-27.

55. Zhou CN, Chao FL, Zhang Y, Jiang L, Zhang L, Luo YM, et al. Sex Differences in the White Matter and Myelinated Fibers of APP/PS1 Mice and the Effects of Running Exercise on the Sex Differences of AD Mice. Frontiers in aging neuroscience. 2018;10:243.

56. Mifflin MA, Winslow W, Surendra L, Tallino S, Vural A, Velazquez R. Sex differences in the IntelliCage and the Morris water maze in the APP/PS1 mouse model of amyloidosis. Neurobiol Aging. 2021;101:130-40.

57. Dominguez S, Rodriguez G, Fazelinia H, Ding H, Spruce L, Seeholzer SH, et al. Sex Differences in the Phosphoproteomic Profiles of APP/PS1 Mice after Chronic Unpredictable Mild Stress. J Alzheimers Dis. 2020;74(4):1131-42.

58. Li X, Feng Y, Wu W, Zhao J, Fu C, Li Y, et al. Sex differences between APPswePS1dE9 mice in A-beta accumulation and pancreatic islet function during the development of Alzheimer's disease. Lab Anim. 2016;50(4):275-85.

59. Mouton-Liger F, Paquet C, Dumurgier J, Bouras C, Pradier L, Gray F, et al. Oxidative stress increases BACE1 protein levels through activation of the PKR-elF2alpha pathway. Biochim Biophys Acta. 2012;1822(6):885-96.

60. Tamagno E, Guglielmotto M, Aragno M, Borghi R, Autelli R, Giliberto L, et al. Oxidative stress activates a positive feedback between the gamma- and beta-secretase cleavages of the beta-amyloid precursor protein. J Neurochem. 2008;104(3):683-95.

61. Mao P, Manczak M, Calkins MJ, Truong Q, Reddy TP, Reddy AP, et al. Mitochondria-targeted catalase reduces abnormal APP processing, amyloid beta production and BACE1 in a mouse model of Alzheimer's disease: implications for neuroprotection and lifespan extension. Hum Mol Genet. 2012;21(13):2973-90. 62. McManus MJ, Murphy MP, Franklin JL. The Mitochondria-Targeted Antioxidant MitoQ Prevents Loss of Spatial Memory Retention and Early Neuropathology in a Transgenic Mouse Model of Alzheimer's Disease. Journal of Neuroscience. 2011;31(44):15703-15. 
63. Adav SS, Park JE, Sze SK. Quantitative profiling brain proteomes revealed mitochondrial dysfunction in Alzheimer's disease. Mol Brain. 2019;12(1):8.

64. Crouch PJ, Barnham KJ, Duce JA, Blake RE, Masters CL, Trounce IA. Copper-dependent inhibition of cytochrome c oxidase by Abeta(1-42) requires reduced methionine at residue 35 of the Abeta peptide. J Neurochem. 2006;99(1):226-36.

\section{Figure Legends}

Figure1: Generation of RISPKO$-A D$ mice. (A) breeding scheme to obtain ClII ${ }^{\mathrm{KO}}-\mathrm{AD}$ animals. (B) Cartoon depicting the induction of Cre recombinase and the consequent excision of exon 2 of UQCRFS1 gene encoding for RISP. (C) PCR products showing the tissue-specific excision of exon 2 of UQCRFS1 gene after tamoxifen induction. (D) qPCR amplifying exon 2 of UQCRFS1 gene, showing that the decrease of the WT allele was comparable in cortex and hippocampus. (E) qPCR amplifying recombined UQCRFS1 gene, showing that the recombination of the excised gene was comparable in cortex and hippocampus.

Figure2: RISP and mitochondrial proteins expression. (A) Western blots of homogenates from cortex (left column) and hippocampus (right column) of 8-month-old females $A D$ and CIIIKO-AD probing for RISP and Core1 (Complex III), NDUFS3 and NDUFB8 (Complex I), SDHA (Complex II), Cyt C and COXI (Complex IV), ATP5A (Complex V) and relative loading controls. (B-C) Quantification of mitochondrial proteins in cortex (B) and hippocampus (C) as a percentage compared to control (indicated as a dashed line). (D-G) Quantification of steady state level of RISP normalized to VDAC1 in cortex (D) and hippocampus (F) and normalized to Core I in cortex and hippocampus $(G)$ of 8-month-old $A D$ and $C I^{K O}-A D$ females. $(H)$ Western blots of homogenates from cortex (left column) and hippocampus (right column) of 8-month-old females $A D$ and CIIIKO-AD probing for mitochondrial membrane proteins VDAC1 and Tim23 and (I) relative quantifications.

Bars represent means \pm SEM of $n=3-4$ for each group, each point is represented by a circle. ${ }^{*} p<0.05$; ${ }^{* *} p<0.01$; ${ }^{* * *} p<0.001$.

Figure3: Induced Complex III deficiency. (A-B) Spectrophotometric Complex III/ Citrate Synthase (CIII/CS) activity ratio $(\mathrm{A})$ and CIV/CS activity ratio $(\mathrm{B})$ measured in cortical homogenates of 8-month-old $\mathrm{AD}$ and $\mathrm{CIII} \mathrm{KO}_{-}$ AD females. (C-D) Spectrophotometric CIII/CS activity ratio (C) and CIV/CS activity ratio (D) measured in hippocampal homogenates of 8-month-old $A D$ and CIIIKO-AD females. (E) Steady state levels of complexes and 
supercomplexes measured by BN-PAGE in homogenates from cortex (left column) and hippocampus (right column) of 8-month-old $A D$ and CIIIKO-AD females. The antibodies used for the different complexes were: NDUFA9 (Complex I), RISP (Complex III), COX1 (Complex IV), ATP5a (Complex V), and SDHA (Complex II). Mitochondrial content was measured by western blot of the same homogenates using antibody against Tim23, VDAC1, and SDHA. (F) Quantification of blue natives from cortical homogenates (F) and hippocampal homogenates (G) normalized for mitochondrial content (Tim23). Loading indicated with \$ is repeated, as Rieske, Corel and VDAC1 were probed on the same gels, therefore share the same loading control.

Bars represent means \pm SEM of $n=3-4$ for each group, each point is represented by a circle. ${ }^{*} p<0.05$; ${ }^{* *} p<0.01$; ${ }^{* * *} p<0.001$

Figure4: Neuronal content and Neuroinflammation: (A) Representative images of IHC probing for neuronal marker NeuN staining on coronal sections of 8-month-old AD and CIII $\mathrm{KO}_{-}$AD females. (B) Western blot probing for Class III $\beta$-Tubulin (TUJ1) and Synaptophysin on cortical and hippocampal homogenates from 8-month-old $A D$ and CIIIKO-AD females (n=3-4/group) and relative quantifications. (C) Representative images of IHC probing for glial marker GFAP on cortex (upper row) and hippocampus (lower row) of 8-month-old AD and CIIIKO-AD females. (D) Western blot probing for glial marker GFAP and microglial marker lba1 on cortical and hippocampal homogenates from 8-month-old $A D$ and $C I^{K O}-A D$ females ( $n=3-4 /$ group) and relative quantifications. ${ }^{*} p<0.05$

Figure5: Amyloid plaques and $A \beta$ content $(A)$ Representative images of coronal sections of $A D$ and $C^{\prime I I K O}$ $A D$ females' brains, immunostained with an anti-A $\beta$ antibody to detect amyloid plaques. (B) Quantification of amyloid plaques in $A D$ and ClIIKO-AD females in cortex and hippocampus ( $n=3 / g r o u p)$. (C) Content of $A \beta 42$ fragments in cortical and hippocampal homogenates from AD and CIIIKO-AD females (n 3-5/group). (D) Western blot analysis detecting the presence of total APP and carboxy-terminal fragment (Ctf) in cortical and hippocampal homogenates from $A D$ and $C I^{K O}{ }_{-} A D$ females; quantification of optical density of Ctf/APP signal from Western blot analysis ( $n=3-4 / g r o u p)$. (E) Enzymatic activity of beta-secretase in cortical homogenates of $A D$ and $C I^{K O_{-}}$ $A D$ females ( $n=4-5 /$ group). (F) Western blot analysis and relative quantification probing for Nicastrin and Presenilin 1 (Psn1) in cortical and hippocampal homogenates from AD and CIIIKO-AD females (n=3-4/group). * $p$ $<0.05$. 
Figure 6: Oxidative stress (A) Western blot analysis and relative quantification probing for SOD2 in cortical and hippocampal homogenates from AD and CIII ${ }^{\mathrm{O}}-\mathrm{AD}$ females (n=3-4/group). (B) Quantification of carbonyl groups added to protein side chains as consequence of oxidative stress (Oxyblot) in cortical and hippocampal homogenates from $A D$ and $C I^{K O}{ }_{-} A D$ females ( $\left.n=3-4 / g r o u p\right)$. (C) Representative images of coronal sections of $A D$ and $C I I{ }^{K O}-A D$ females' brains, immunostained with an anti-8OHg antibody to detect DNA/RNA oxidation. In the close-up: positive control $\left(\mathrm{H}_{2} \mathrm{O}_{2}\right.$ treatment) and negative control (DNAse treatment). ${ }^{*} p<0.05$.

Suppl. Figure1: RISP and mitochondrial proteins expression in males. (A) Western blots of homogenates from cortex (left column) and hippocampus (right column) of 8-month-old males $A D$ and $\mathrm{CIII}^{\mathrm{KO}}-\mathrm{AD}$ probing for RISP and Core1 (Complex III), NDUFS3 and NDUFB8 (complex I), SDHA (complex II), Cyt C and COXI (Complex IV) and relative loading controls. (B-C) Quantification of mitochondrial proteins in cortex (B) and hippocampus (C) as a percentage compared to control (indicated as a dashed line). (D-G) Quantification of steady state level of RISP normalized to VDAC1 in cortex (D) and hippocampus (F) and normalized to Core1 in cortex (E) and hippocampus $(\mathrm{G})$ of 8-month-old $A D$ and $C I^{K O}-A D$ males. $(H)$ Western blots of homogenates from cortex (left column) and hippocampus (right column) of 8-month-old males $A D$ and $C \mathrm{II}^{\mathrm{KO}}-\mathrm{AD}$ probing for mitochondrial membrane proteins VDAC1 and Tim23 and (I) relative quantifications. Loading indicated with $\$$ is repeated, as Rieske, Corel and VDAC1 were probed on the same gels, therefore share the same loading control. Bars represent means \pm SEM. $n=3-4 /$ group, each point is represented by a circle. ${ }^{*} p<0.05 ;{ }^{* *} p<0.01$.

Suppl. Figure2: Complex III deficiency. Spectrophotometric Complex III, Complex IV and Citrate synthase (CS) activity normalized to protein loading, measured in cortical and hippocampal homogenates of 8-month-old $A D$ and CIIIKO-AD females. Bars represent means \pm SEM. $n=3-4 /$ group, each point is represented by a circle. * $p<$ $0.05 ;{ }^{* *} p<0.01$

Suppl. Figure3: Neuronal content and Neuroinflammation in males (A) Representative images of H\&E staining on histological sections from $A D$ and $C I I{ }^{K O}-A D$ females and males. (B) Representative images of Nissl staining on histological sections from $A D$ and $C I I{ }^{K O}-A D$ females and males. (C) Representative images of IHC 
probing for neuronal marker NeuN staining on coronal sections of 8-month-old ClIIKO-AD and AD males. (D) Western blot probing for Class III $\beta$-Tubulin (TUJ1) and Synaptophysin on cortical and hippocampal homogenates from 8-month-old $C \mathrm{ClI}^{\mathrm{KO}}-\mathrm{AD}$ and $\mathrm{AD}$ males (n=3-4/group) and relative quantifications. (E) Representative images of IHC probing for glial marker GFAP on cortex (upper row) and hippocampus (lower row) of 8-month-old CIIIKO-AD and AD males. (F) Western blot probing for glial marker GFAP and microglial marker Iba1 on cortical and hippocampal homogenates from 8-month-old CIIIKO-AD and AD males (n=3-4/group) and relative quantifications. ${ }^{*} p<0.05$

Suppl. Figure4: proteins involved in degradation pathways. (A) Western blots of homogenates from cortex and hippocampus of 8-month-old females $A D$ and $\mathrm{CIIIKO}_{-} \mathrm{AD}$ probing for $\mathrm{p62}$ and LC3B I-II and relative quantification ( $n=3-4 /$ group). (B) Western blots of homogenates from cortex and hippocampus of 8-month-old females $A D$ and $C I^{K O}-A D$ probing for 20 s proteasome and relative quantification ( $n=3-4 /$ group).

Suppl. Figure5: Amyloid plaques and $A \beta$ content in males (A) Representative images of coronal sections of $A D$ and $C I I K^{K O}-A D$ males' brains, immunostained with an anti-A $\beta$ antibody to detect amyloid plaques. (B) Quantification of amyloid plaques in AD and CIIIKO-AD males in cortex and hippocampus ( $n=3 /$ group). (C) Content of $A \beta 42$ fragments in cortical and hippocampal homogenates from $A D$ and $C I^{K O}-A D$ males ( $\left.n=3-5 / g r o u p\right)$. (D) Western blot analysis detecting the presence of total APP and carboxy-terminal fragment (Ctf) in cortical and hippocampal homogenates from $A D$ and $C I^{K O_{-}} A D$ males; quantification of optical density of Ctf/APP signal from Western blot analysis ( $n=3-4 /$ group). 

Figures

A

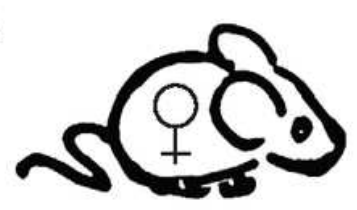

RISPFW

CamKIla-CRE ERT ${ }^{+/}$

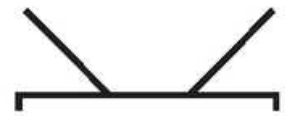

CIII ${ }^{\mathrm{KO}}-\mathrm{AD}$

$A D$

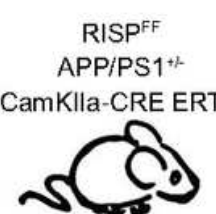

C

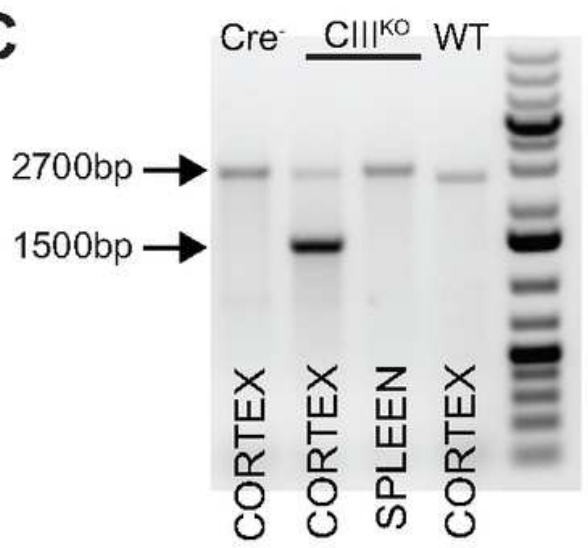

$$
\begin{gathered}
\text { RISPF } \\
\text { APP/PS1 } \\
\text { */- }
\end{gathered}
$$

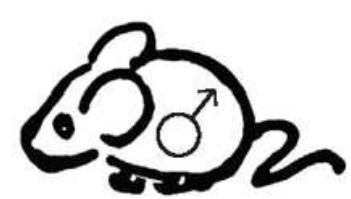

RISPFW APP/PS1 ${ }^{+1-}$

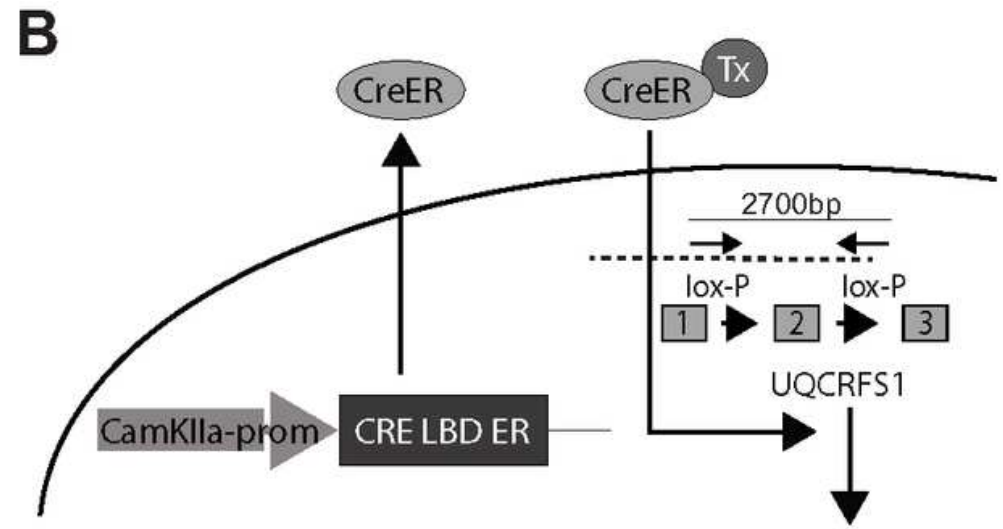

$1500 \mathrm{bp}$
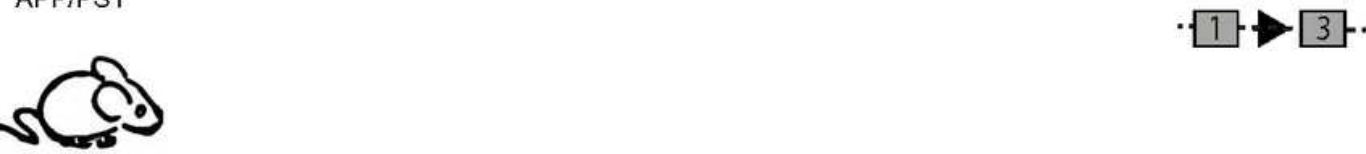

D
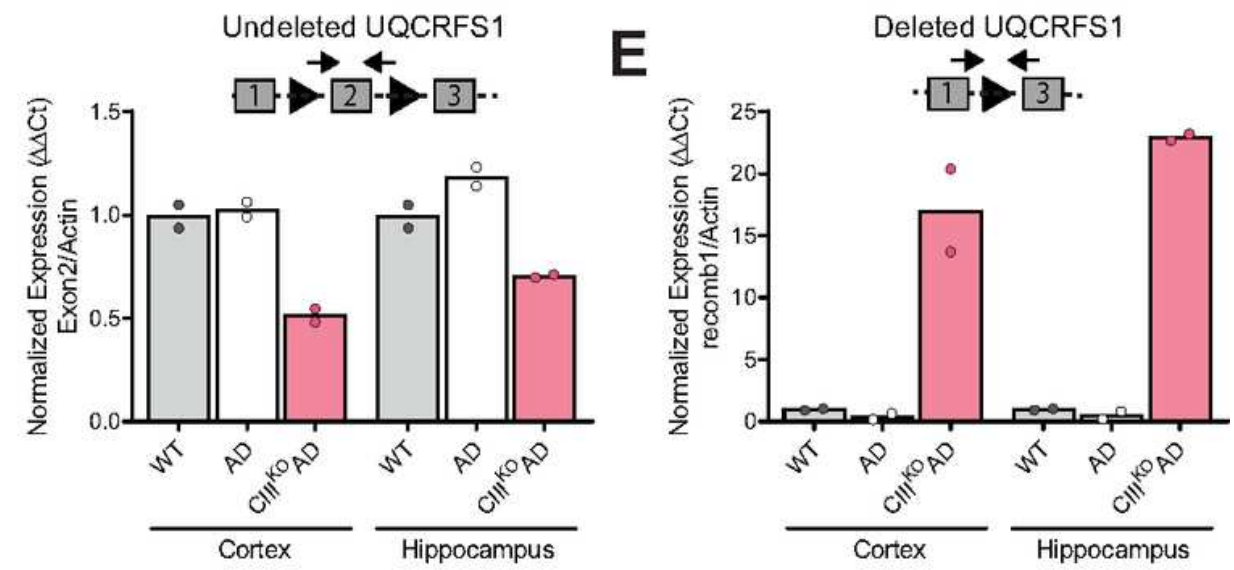

\section{Figure 1}

Figure 1

Generation of RISPKO-AD mice. (A) breeding scheme to obtain CIIIKO-AD animals. (B) Cartoon depicting the induction of Cre recombinase and the consequent excision of exon 2 of UQCRFS1 gene encoding for RISP. (C) PCR products showing the tissue-specific excision of exon 2 of UQCRFS1 gene after tamoxifen induction. (D) qPCR amplifying exon 2 of UQCRFS1 gene, showing that the decrease of the WT allele was comparable in cortex and hippocampus. (E) qPCR amplifying recombined UQCRFS1 gene, showing that the recombination of the excised gene was comparable in cortex and hippocampus. 

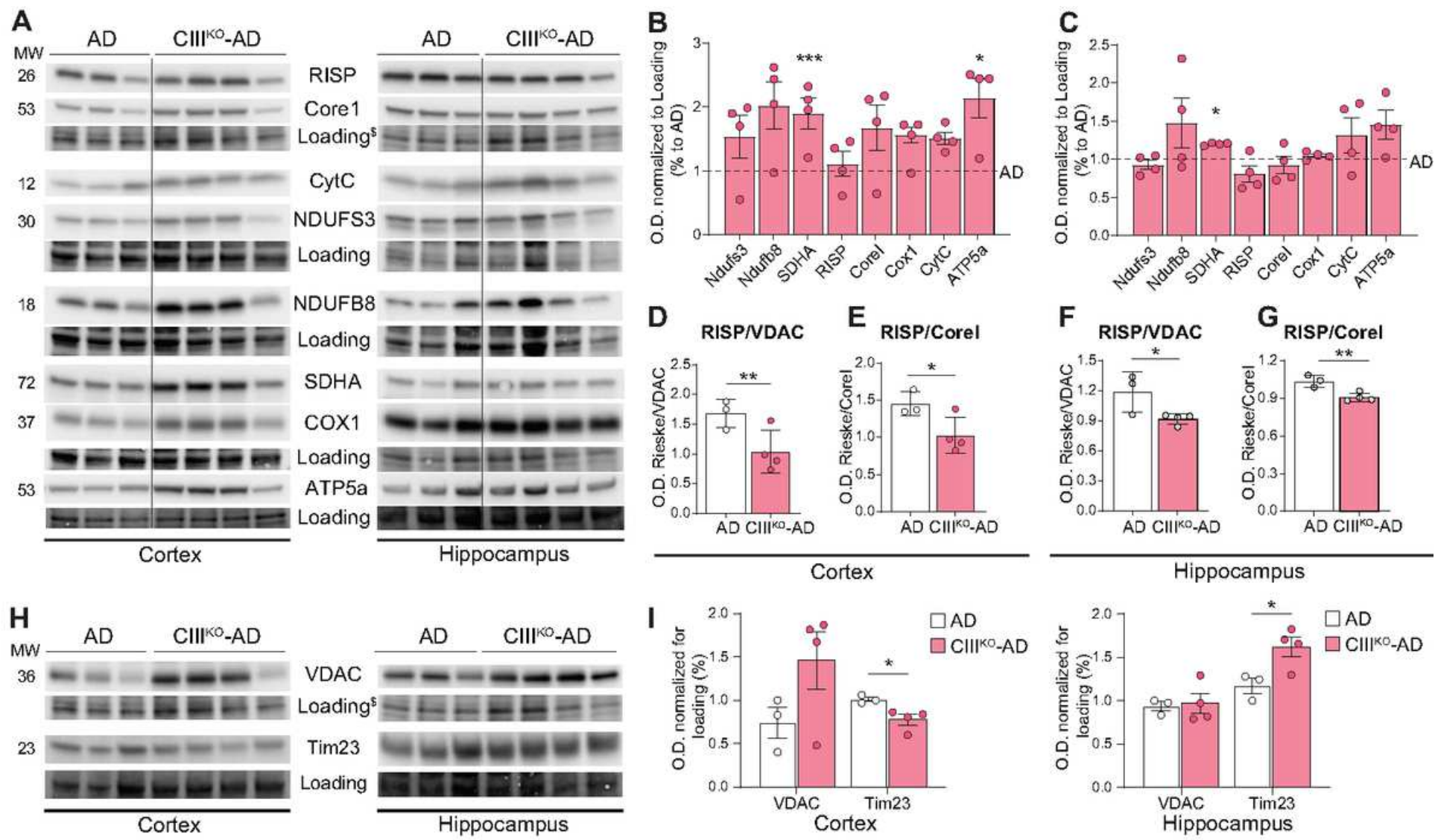

Hippocampus

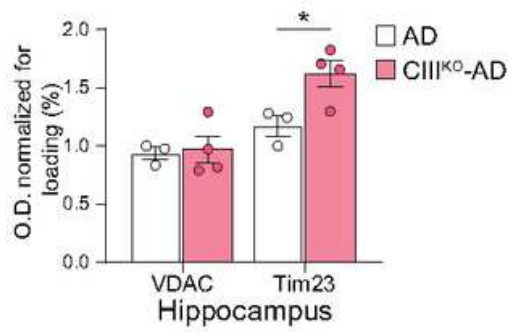

Figure 2

\section{Figure 2}

RISP and mitochondrial proteins expression. (A) Western blots of homogenates from cortex (left column) and hippocampus (right column) of 8-month-old females AD and CIIIKO-AD probing for RISP and Core1 (Complex III), NDUFS3 and NDUFB8 (Complex I), SDHA (Complex II), Cyt C and COXI (Complex IV), ATP5A (Complex V) and relative loading controls. (B-C) Quantification of mitochondrial proteins in cortex (B) and hippocampus (C) as a percentage compared to control (indicated as a dashed line). (D-G) Quantification of steady state level of RISP normalized to VDAC1 in cortex (D) and hippocampus (F) and normalized to Core I in cortex and hippocampus (G) of 8-month-old AD and CIIIKO-AD females. (H) Western blots of homogenates from cortex (left column) and hippocampus (right column) of 8-month-old females $A D$ and CIIIKO-AD probing for mitochondrial membrane proteins VDAC1 and Tim23 and (I) relative quantifications. Bars represent means \pm SEM of $n=3-4$ for each group, each point is represented by a circle. ${ }^{\star} p<0.05 ;{ }^{* \star} p<0.01 ;{ }^{* \star *} \mathrm{p}<0.001$. 
A

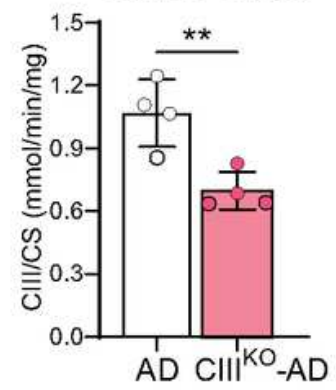

E

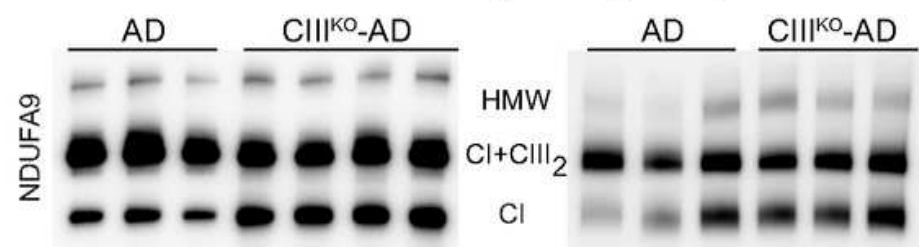

HMW

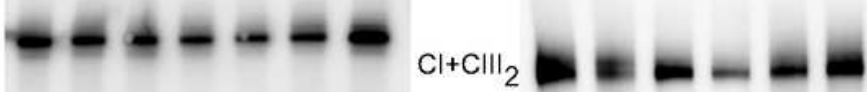

$\frac{0}{\frac{0}{x}}$

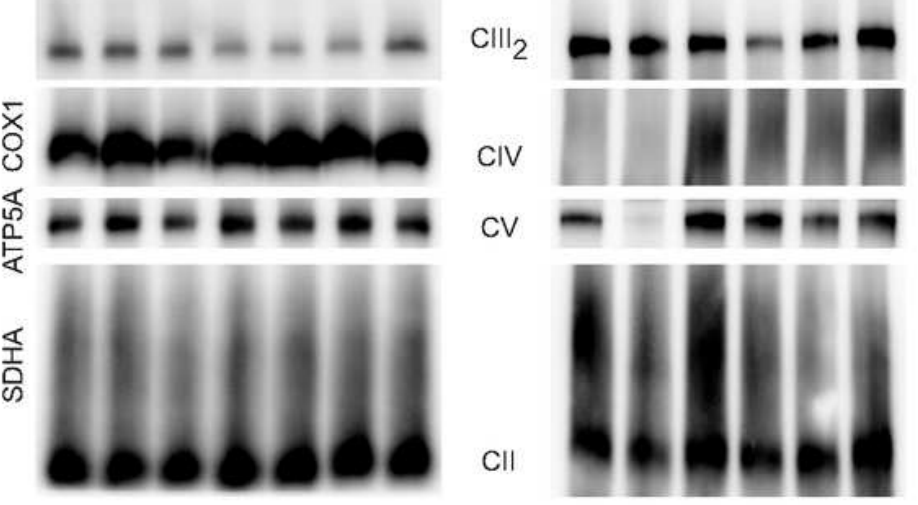

SDS-PAGE

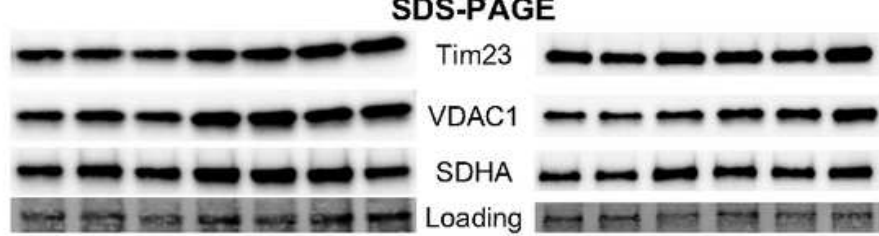

Cortex

Hippocampus

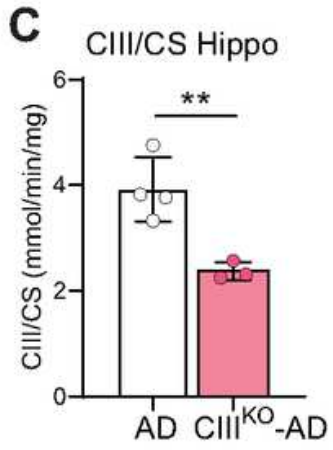

D CIVICS Hippo
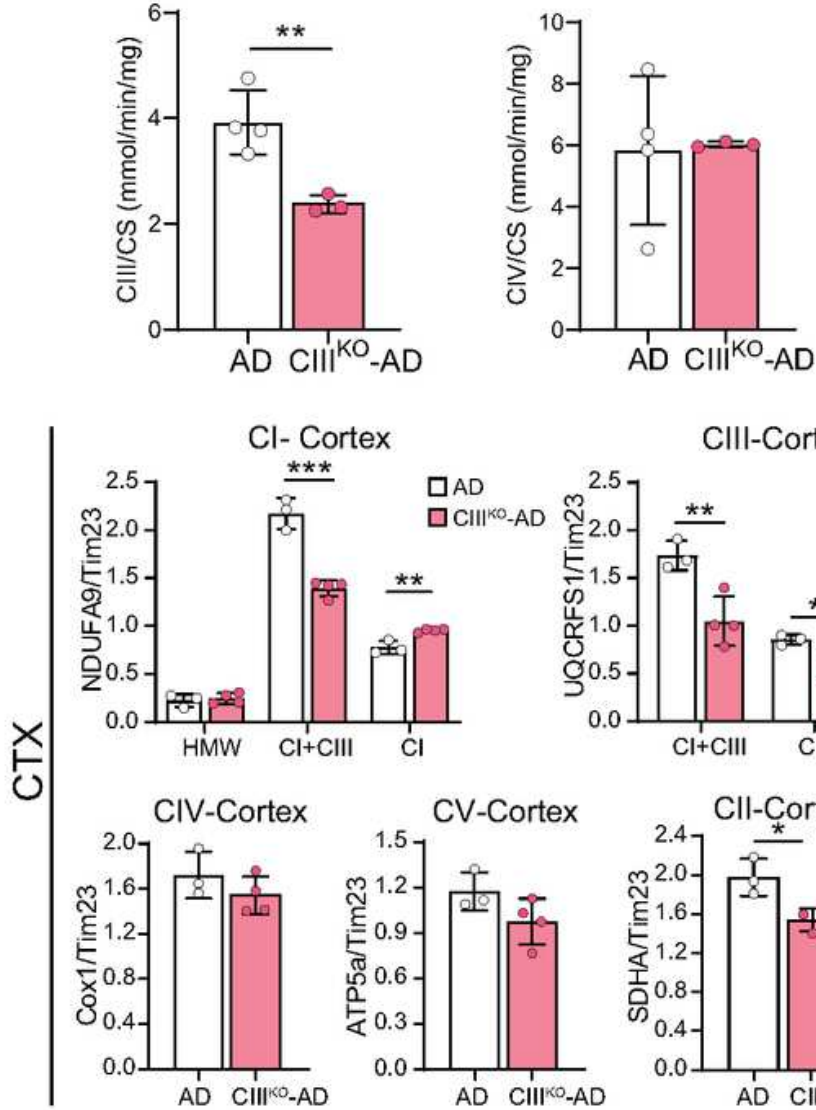

- Cortex

CIII-Cortex

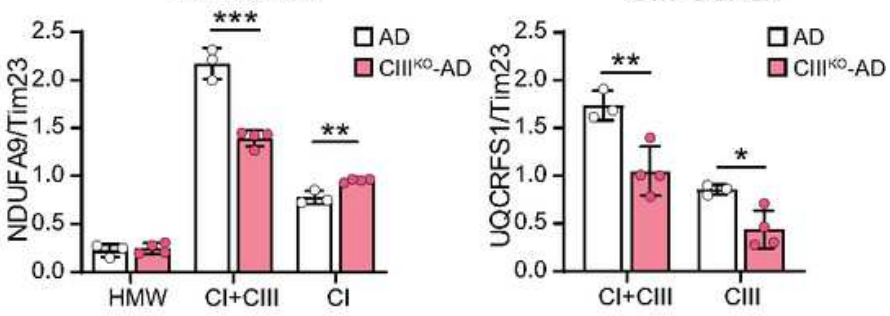

포
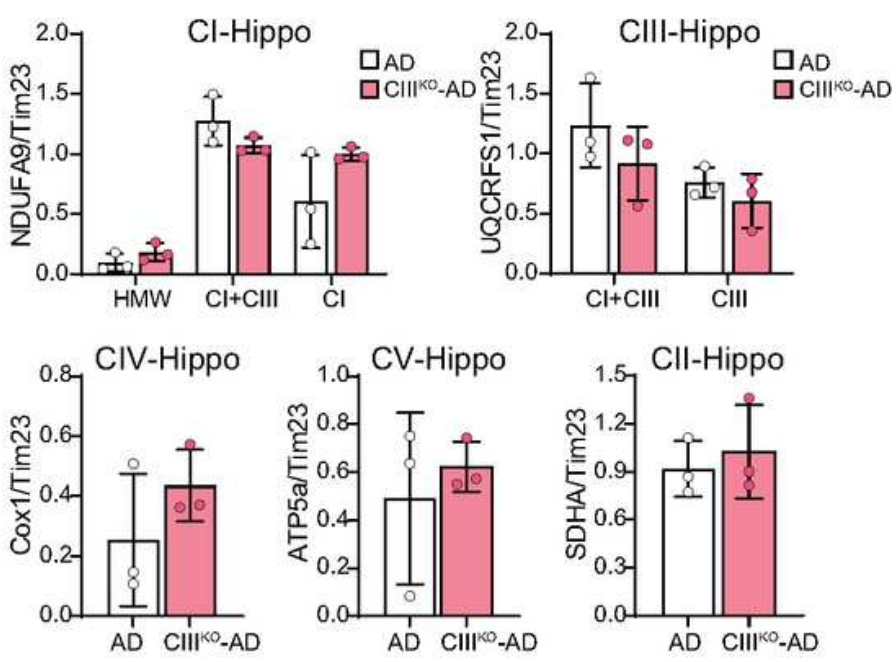

Figure 3

\section{Figure 3}

Induced Complex III deficiency. (A-B) Spectrophotometric Complex III/ Citrate Synthase (CIII/CS) activity ratio (A) and CIV/CS activity ratio (B) measured in cortical homogenates of 8-month-old AD and CIIIKOAD females. (C-D) Spectrophotometric CIII/CS activity ratio (C) and CIV/CS activity ratio (D) measured in hippocampal homogenates of 8-month-old $A D$ and CIIIKO-AD females. (E) Steady state levels of complexes and supercomplexes measured by BN-PAGE in homogenates from cortex (left column) and hippocampus (right column) of 8-month-old AD and CIIIKO-AD females. The antibodies used for the 
different complexes were: NDUFA9 (Complex I), RISP (Complex III), COX1 (Complex IV), ATP5a (Complex $\mathrm{V})$, and SDHA (Complex II). Mitochondrial content was measured by western blot of the same homogenates using antibody against Tim23, VDAC1, and SDHA. (F) Quantification of blue natives from cortical homogenates $(F)$ and hippocampal homogenates $(G)$ normalized for mitochondrial content (Tim23). Loading indicated with $\$$ is repeated, as Rieske, Corel and VDAC1 were probed on the same gels, therefore share the same loading control. Bars represent means $\pm S E M$ of $n=3-4$ for each group, each point is represented by a circle. ${ }^{*} p<0.05 ;{ }^{* *} p<0.01 ; * \star * p<0.001$.

A
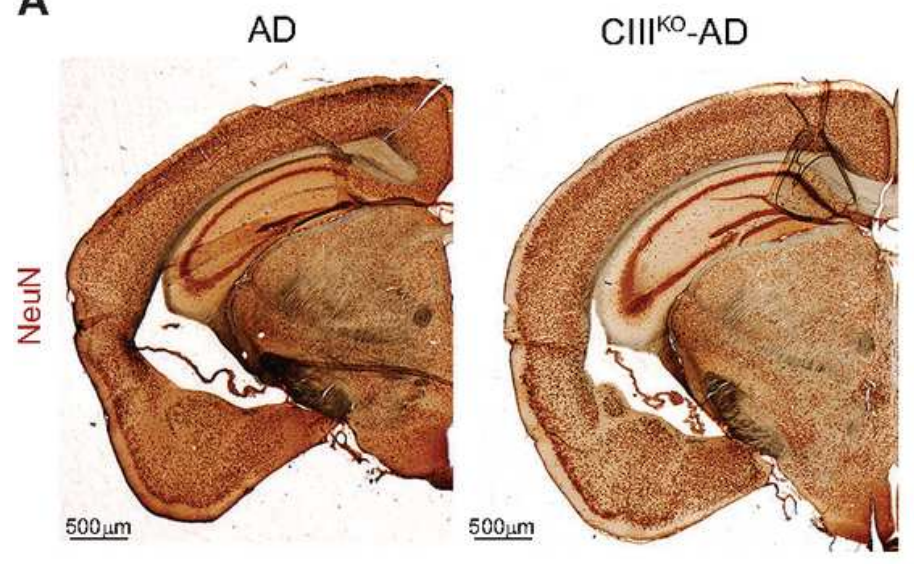

C

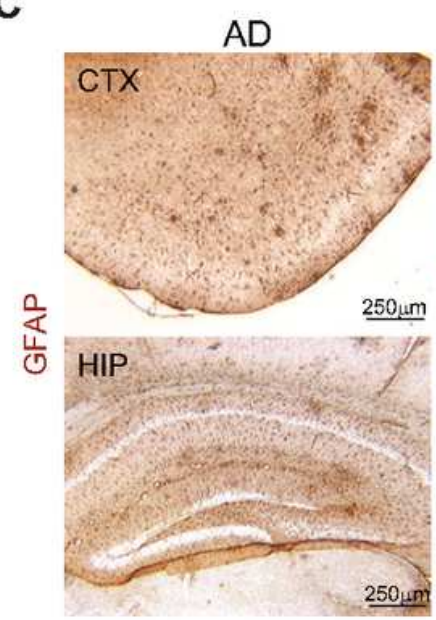

Figure 4

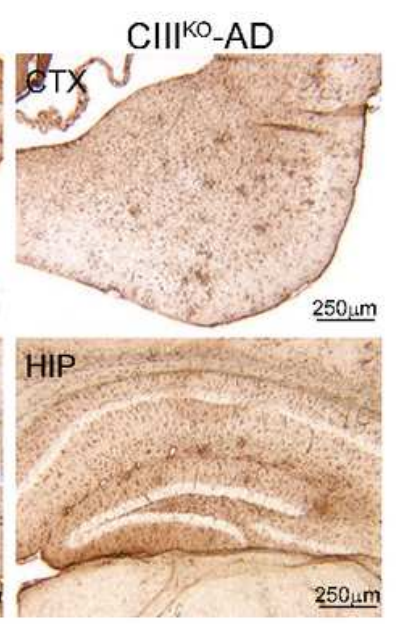

B
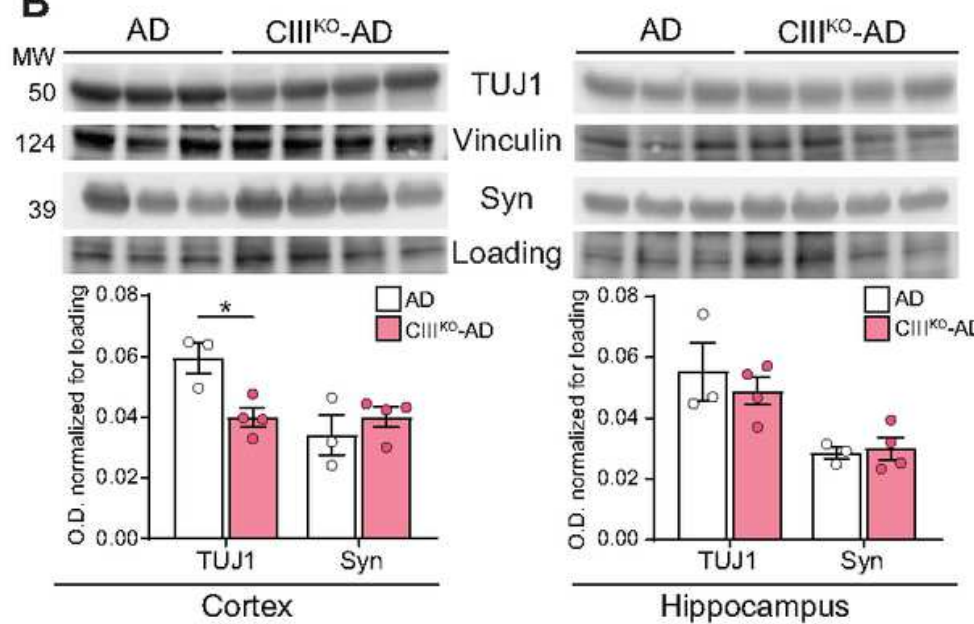

D
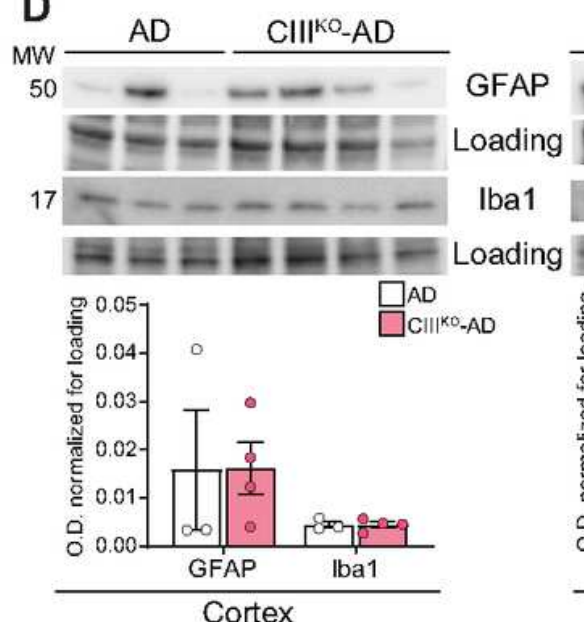

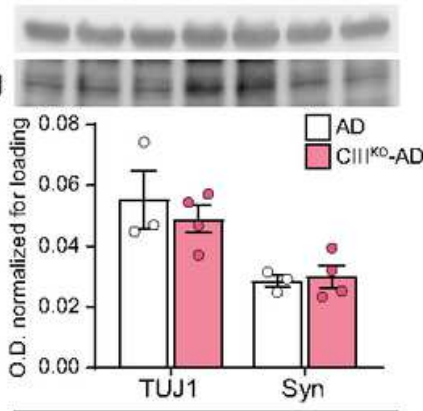

Hippocampus
Cortex

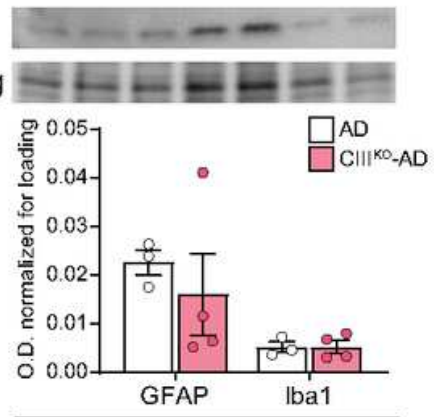

Hippocampus

\section{Figure 4}

Neuronal content and Neuroinflammation: (A) Representative images of IHC probing for neuronal marker NeuN staining on coronal sections of 8-month-old AD and CIIIKO-AD females. (B) Western blot probing for Class III $\beta$-Tubulin (TUJ1) and Synaptophysin on cortical and hippocampal homogenates from 8-monthold AD and CIIIKO-AD females ( $n=3-4 /$ group) and relative quantifications. (C) Representative images of IHC probing for glial marker GFAP on cortex (upper row) and hippocampus (lower row) of 8-month-old AD and CIIIKO-AD females. (D) Western blot probing for glial marker GFAP and microglial marker Iba1 on cortical and hippocampal homogenates from 8-month-old AD and CIIIKO-AD females ( $n=3-4 /$ group) and relative quantifications. ${ }^{*} p<0.05$ 
A

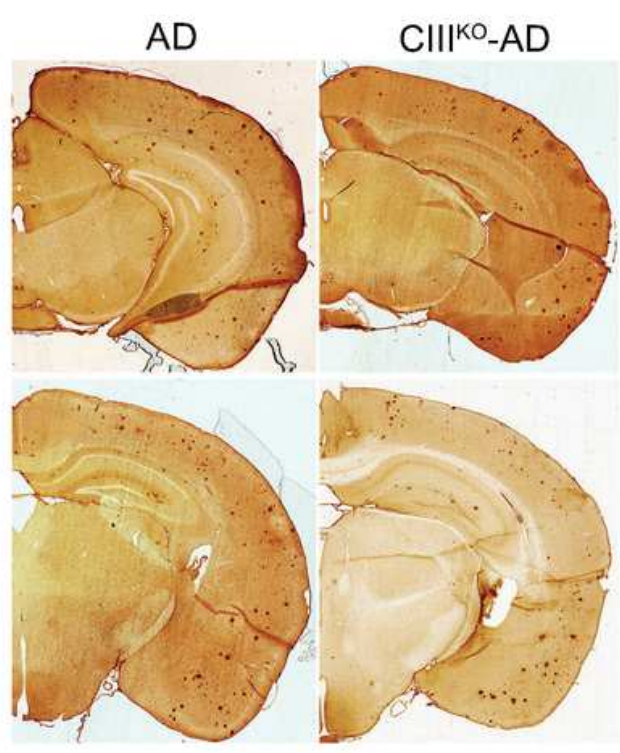

B

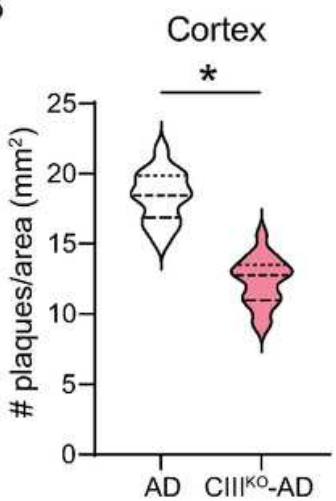

Hippocampus

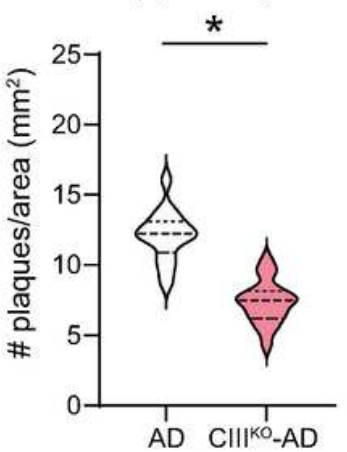

E

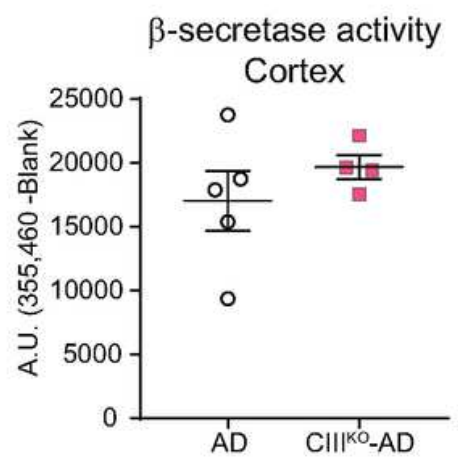

C

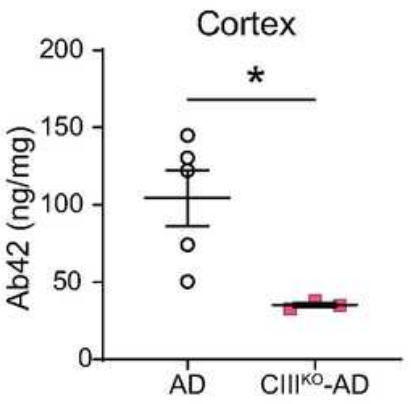

D
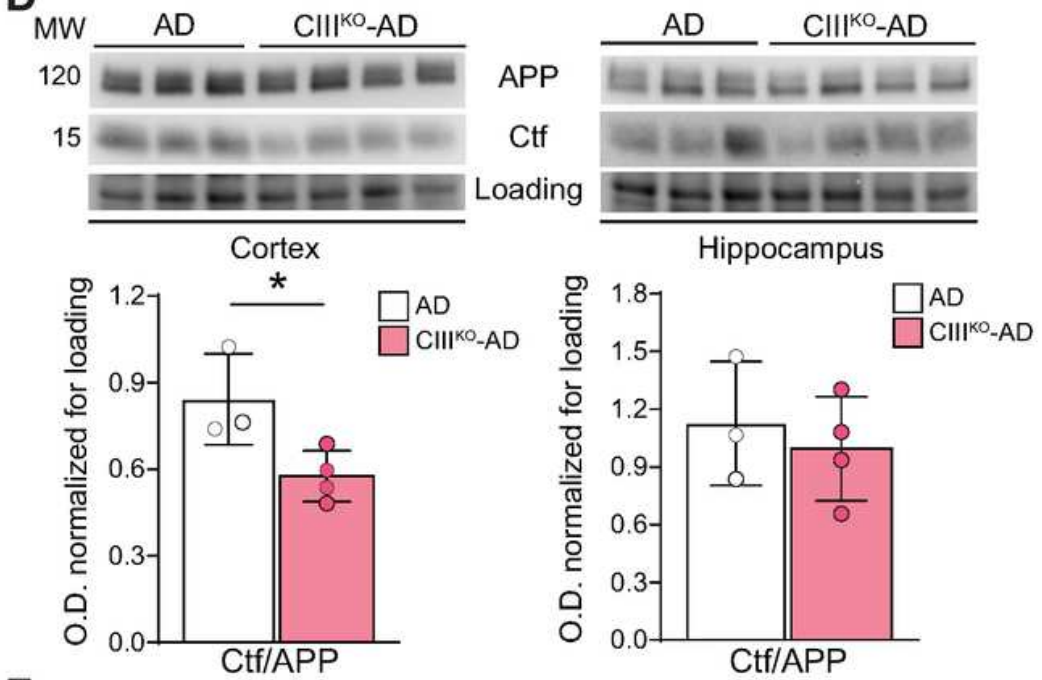

F
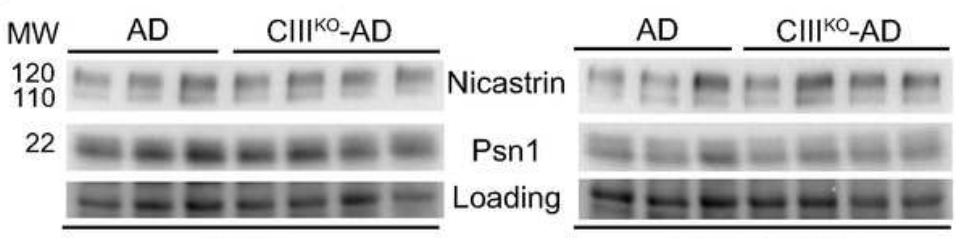

Cortex

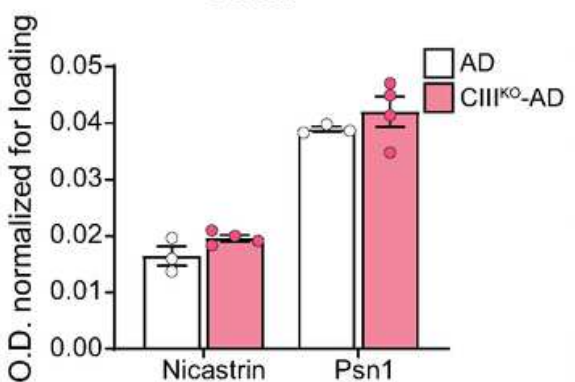

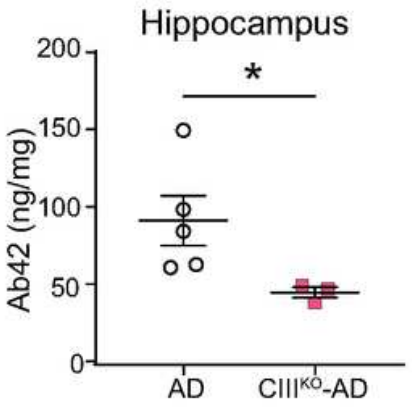

AD

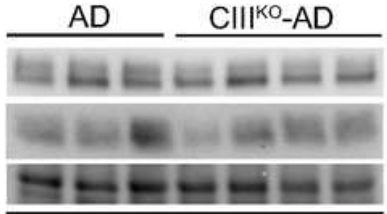

Hippocampus

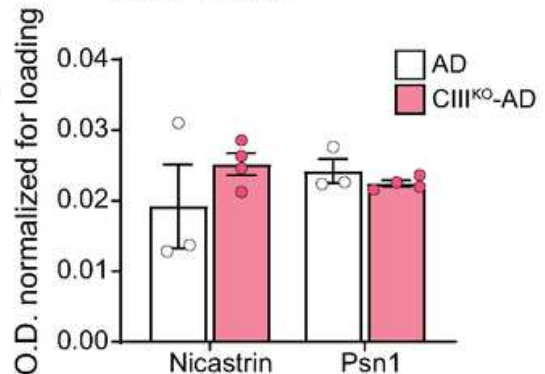

Figure 5

\section{Figure 5}

Amyloid plaques and $A \beta$ content $(A)$ Representative images of coronal sections of $A D$ and $C$ IIIKO-AD females' brains, immunostained with an anti-A $\beta$ antibody to detect amyloid plaques. (B) Quantification of amyloid plaques in AD and CIIIKO-AD females in cortex and hippocampus ( $n=3 /$ group). (C) Content of $A \beta 42$ fragments in cortical and hippocampal homogenates from $A D$ and CIIIKO-AD females (n 35/group). (D) Western blot analysis detecting the presence of total APP and carboxy-terminal fragment (Ctf) in cortical and hippocampal homogenates from $A D$ and CIIIKO-AD females; quantification of optical density of Ctf/APP signal from Western blot analysis (n=3-4/group). (E) Enzymatic activity of beta- 
secretase in cortical homogenates of $A D$ and CIIIKO-AD females ( $n=4-5 /$ group). (F) Western blot analysis and relative quantification probing for Nicastrin and Presenilin 1 (Psn1) in cortical and hippocampal homogenates from AD and CIIIKO-AD females (n=3-4/group). * $p<0.05$.
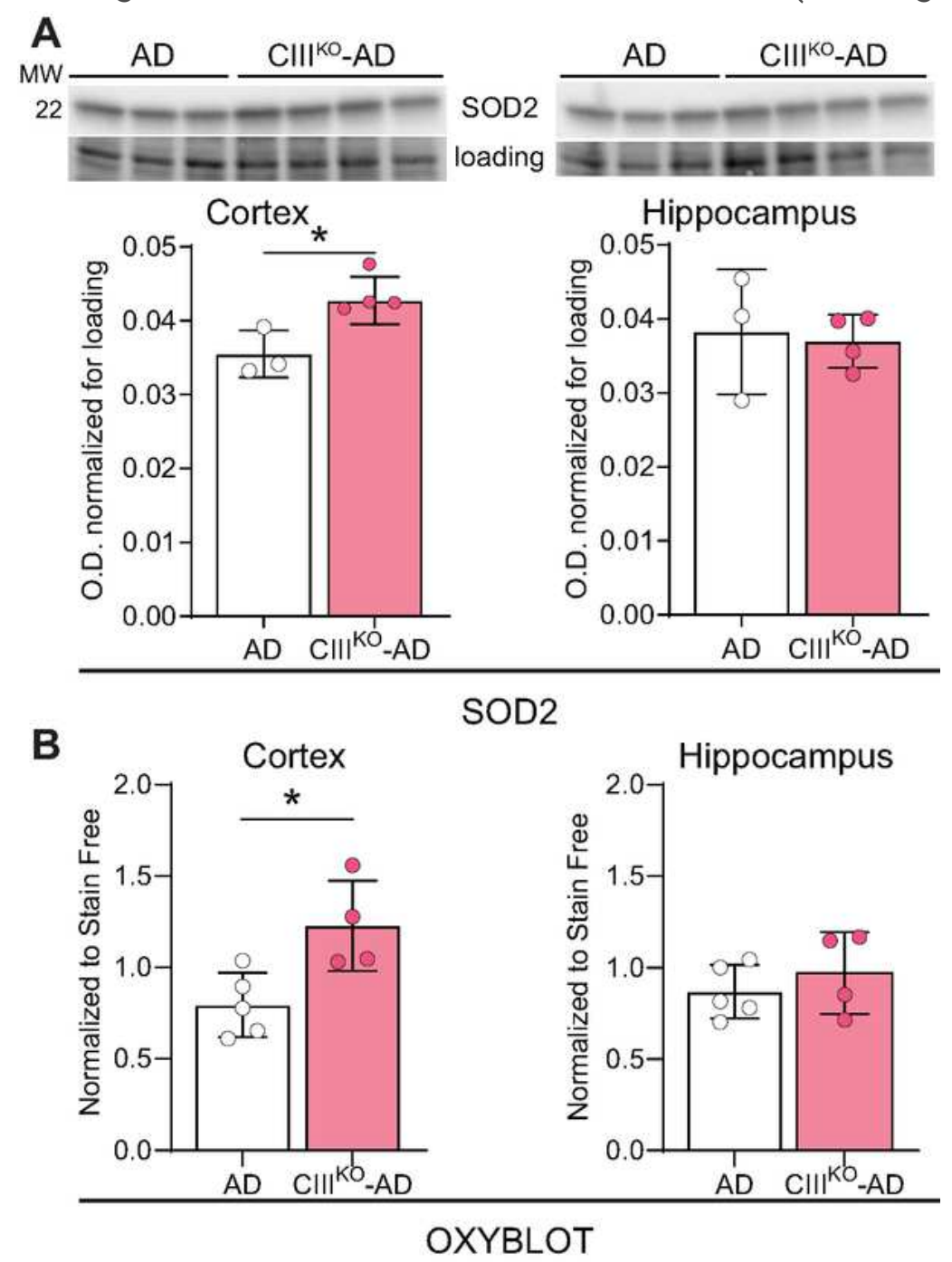

SOD2

C

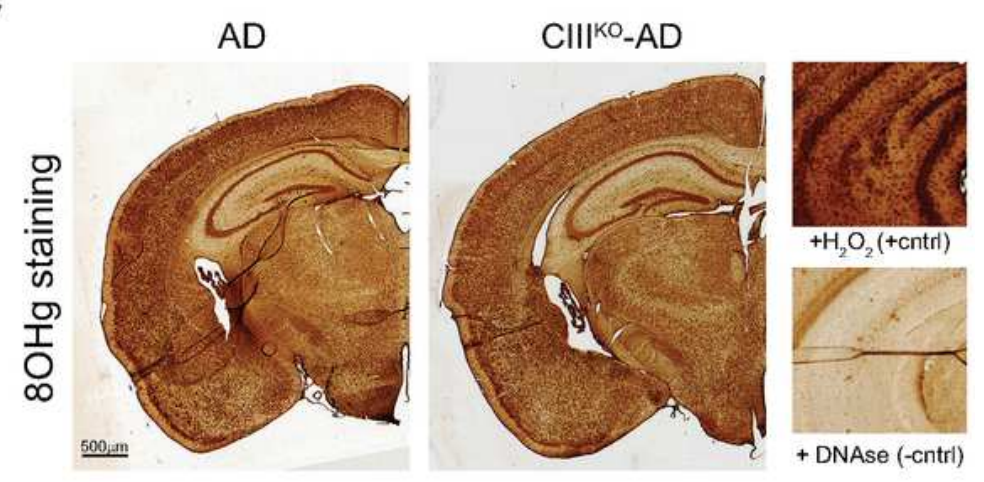

Figure 6

Figure 6

Oxidative stress (A) Western blot analysis and relative quantification probing for SOD2 in cortical and hippocampal homogenates from AD and CIIIKO-AD females ( $n=3-4 /$ group). (B) Quantification of carbonyl 
groups added to protein side chains as consequence of oxidative stress (Oxyblot) in cortical and hippocampal homogenates from AD and CIIIKO-AD females (n=3-4/group). (C) Representative images of coronal sections of AD and CIIIKO-AD females' brains, immunostained with an anti-8OHg antibody to detect DNA/RNA oxidation. In the close-up: positive control (H2O2 treatment) and negative control (DNAse treatment). ${ }^{*} p<0.05$.

\section{Supplementary Files}

This is a list of supplementary files associated with this preprint. Click to download.

- Pintoetal.SupplementaryFigures.pdf 